\title{
REDUNDANT DECOMPOSITIONS, ANGLES BETWEEN SUBSPACES AND OBLIQUE PROJECTIONS
}

\author{
G. Corach and A. Maestripieri
}

\begin{abstract}
Let $\mathcal{H}$ be a complex Hilbert space. We study the relationships between the angles between closed subspaces of $\mathcal{H}$, the oblique projections associated to non direct decompositions of $\mathcal{H}$ and a notion of compatibility between a positive (semidefinite) operator $A$ acting on $\mathcal{H}$ and a closed subspace $\mathcal{S}$ of $\mathcal{H}$. It turns out that the compatibility is ruled by the values of the Dixmier angle between the orthogonal complement $\mathcal{S}^{\perp}$ of $\mathcal{S}$ and the closure of $A \mathcal{S}$. We show that every redundant decomposition $\mathcal{H}=\mathcal{S}+\mathcal{M}^{\perp}$ (where redundant means that $\mathcal{S} \cap \mathcal{M}^{\perp}$ is not trivial) occurs in the presence of a certain compatibility. We also show applications of these results to some signal processing problems (consistent reconstruction) and to abstract splines problems which come from approximation theory.
\end{abstract}

\section{Introduction}

Let $\mathcal{H}$ be a Hilbert space, $L(\mathcal{H})$ the algebra of bounded linear operators on $\mathcal{H}$ and $L(\mathcal{H})^{+}$the convex cone of all positive semidefinite operators in $L(\mathcal{H})$; in this paper, "positive" means "semidefinite positive". A closed subspace $\mathcal{S}$ of $\mathcal{H}$ and $A \in L(\mathcal{H})^{+}$are called compatible if there exists a projection $Q \in L(\mathcal{H})$ with image $\mathcal{S}$ such that $A Q=Q^{*} A$. The notion of compatibility between subspaces and positive operators has been introduced in [13] and applied to abstract splines in [14]. Related notions have been studied earlier by Hassi and Nordström [25]. We recently noticed [12] that Sard considered a similar notion in 1952 in his studies on approximation processes [38]. Sard's idea received little attention, perhaps because the concept of ill-conditioned matrices (operators) was not still fully developed. In statistics, inner products are

2000 Mathematics Subject Classification. 46C05, 47A62, 94A12, 41A65.

Key words. Oblique projections, angles between subspaces, compatibility, abstract splines.

Partially supported by FONCYT PICT 808-2008 and UBACYT I023. 
frequently defined by certain correlation matrices, which are supposed to be positive definite. The orthogonal projection matrices, with respect to these inner products, are extensively treated in the text by Harville [24]. For an infinite dimensional Hilbert space $\mathcal{H}$, Pasternak-Winiarski [35] studied the analytical dependence on $A$ of the projection $P_{A, \mathcal{S}}$ onto a closed subspace $\mathcal{S}$, which is orthogonal with respect to the inner product defined by the invertible operator $A$. In [2], Pasternak-Winiarski's results were extended to the case where also $\mathcal{S}$ varies. This provides a well-defined map $G L(\mathcal{H})^{+} \times G r(\mathcal{H}) \rightarrow \mathcal{Q}$, where $G L(\mathcal{H})^{+}$denotes the set of positive invertible operators in $\mathcal{H}, G r(\mathcal{H})$ is the Grassmannian of $\mathcal{H}$ and $\mathcal{Q}$ is the set of all bounded linear projections on $\mathcal{H}$. If, for a fixed $\mathcal{S} \in \operatorname{Gr}(\mathcal{H}), \mathcal{Q}_{\mathcal{S}}$ denotes the set of all $Q \in \mathcal{Q}$ such that its image $R(Q)$ is $\mathcal{S}$, then $P_{A, \mathcal{S}} \in \mathcal{Q}_{\mathcal{S}}$. One of the goals in [13] was the extension of the map $(A, \mathcal{S}) \rightarrow P_{A, \mathcal{S}}$ allowing $A$ to be non invertible. However, there is no such map $L(\mathcal{H})^{+} \times G r(\mathcal{H}) \rightarrow \mathcal{Q}$ as before. As we said, a pair $(A, \mathcal{S}) \in L(\mathcal{H})^{+} \times G r(\mathcal{H})$ is called compatible if there exists $Q \in \mathcal{Q}_{\mathcal{S}}$ such that $A Q=Q^{*} A$. This means that $\langle Q h, k\rangle_{A}=\langle h, Q k\rangle_{A}$ for all $h, k \in \mathcal{H}$, where $\langle h, k\rangle_{A}=\langle A h, k\rangle$. We show that, if $(A, \mathcal{S})$ is compatible, then there exists a particular $P_{A, \mathcal{S}} \in \mathcal{Q}_{\mathcal{S}}$ which is $A$-orthogonal. The domain of the map $(A, \mathcal{S}) \rightarrow P_{A, \mathcal{S}}$ is the set of all compatible pairs. It is a set which lies strictly between $G L(\mathcal{H})^{+} \times G r(\mathcal{H})$ and $L(\mathcal{H})^{+} \times G r(\mathcal{H})$. One of the goals of this paper is to characterize compatible pairs in terms of Dixmier's angles. Recall that, given closed subspaces $\mathcal{S}_{1}$ and $\mathcal{S}_{2}$, the Dixmier angle between $\mathcal{S}_{1}$ and $\mathcal{S}_{2}$ is that $\alpha \in[0, \pi / 2]$ such that $\cos \alpha=\sup \left\{\left|\left\langle s_{1}, s_{2}\right\rangle\right|: s_{1} \in \mathcal{S}_{1}, s_{2} \in \mathcal{S}_{2},\left\|s_{1}\right\|=\left\|s_{2}\right\|=1\right\}$. This concept is also related to non direct decompositions $\mathcal{H}=\mathcal{S}_{1}+\mathcal{S}_{2}$, i.e., such that $\mathcal{S}_{1} \cap \mathcal{S}_{2}$ is not trivial. These decompositions, which are relevant in compatibility theory, have received much attention in linear reconstruction problems from signal processing. A previous application of compatibility to reconstruction problems appeared in $[\mathbf{3}]$. Another goal of the paper is to make explicit the relationship between redundant decompositions of $\mathcal{H}$ and certain compatibility conditions. As an application, we show that many problems like those studied by Unser and Aldroubi [40], Eldar [20] and Hirabayashi and Unser [27] can be extended to infinite dimensional settings in the presence of compatibility. The same type of extension holds for abstract splines problems $[\mathbf{5}],[\mathbf{9}],[\mathbf{2 8}],[\mathbf{1 1}],[\mathbf{1 6}]$ which we describe below. We show that the usual hypothesis are stronger than a compatibility condition, which allows one to solve several problems on interpolating and smoothing splines. It should be remarked that Shekhtman [39], de Boor [9], Izumino [28] and Deutsch [16] also use angles between subspaces in abstract splines problems. 
We briefly describe the contents of the paper. Section 2 surveys some results on two notions by Friedrichs [21] and Dixmier [18] of angles between closed subspaces (see definitions in Section 2). We use these angles to describe decompositions of $\mathcal{H}$ as a sum of two closed subspaces: given subspaces $\mathcal{S}_{1}, \mathcal{S}_{2}$ it holds $\mathcal{H}=\mathcal{S}_{1}+\mathcal{S}_{2}$ if and only if the Dixmier angle between their orthogonal complements is non zero; moreover, $\mathcal{H}=\mathcal{S}_{1} \dot{+} \mathcal{S}_{2}$ if and only if, in addition, the Dixmier angle between $\mathcal{S}_{1}$ and $\mathcal{S}_{2}$ is non zero. In Section 3 we introduce the notion of compatibility. Using the results of Section 2 we prove that the existence of such projections is equivalent to the fact that $\mathcal{S}^{\perp}$ and $A \mathcal{S}$ have non-zero Dixmier angle and that the redundant decompositions of Section 2 are indeed manifestations of compatibility. We include here an application to reconstruction problems of signals, using the notion of consistent reconstruction defined by M. Unser and A. Aldroubi [40]. Section 4 contains different descriptions of the set $\mathcal{P}(A, \mathcal{S})$ of all projections $Q \in \mathcal{Q}_{\mathcal{S}}$, such that $A Q=Q^{*} A$, in the case where $\mathcal{S}$ and $A$ are compatible. It is remarked that in this case there exists a distinguished projection $P_{A, \mathcal{S}} \in \mathcal{P}(A, \mathcal{S})$ with several optimal properties, which are analogous to those of the classical orthogonal projection $P_{\mathcal{S}}$ in the set of all projections with image $\mathcal{S}$. This section contains an extension of a construction, due to Minamide and Nakamura, of a restricted pseudoinverse of an operator $T$ with closed range to a closed subspace $\mathcal{S}$ such that $T \mathcal{S}$ is closed. Section 5 is devoted to study the minimality properties of $P_{A, \mathcal{S}}$ mentioned above. These properties show that $P_{A, \mathcal{S}}$, in many senses, plays the role of the classical orthogonal projection $P_{\mathcal{S}}$. Finally, Section 6 contains several results and applications to abstract spline problems. Atteia [5], [6] introduced the subject and obtained the main results. He got, by Hilbert space methods, a unified approach to the study of different kinds of splines. Essentially, one has operators $T \in L(\mathcal{H}, \mathcal{E}), V \in L(\mathcal{H}, \mathcal{F})$ such that $R(T)$ is closed and $R(V)=\mathcal{F}$. The problem of interpolating abstract splines is to minimize $\|T h\|$, subject to $V h=f_{0}$, for a given $f_{0} \in \mathcal{F}$. The problem of smoothing abstract splines is to minimize $\|T h\|+\rho\left\|V h-f_{0}\right\|$ with no constraint, where $f_{0} \in \mathcal{F}$ and $\rho>0$. Atteia solved these problems with the hypothesis above together with the condition that $T N(V)$ is closed. We extend his results replacing the closedness of $R(T)$ and $T N(V)$ by the compatibility of the nullspace $N(V)$ and $A=T^{*} T$. This hypothesis is strictly weaker than Atteia's. We also show that there exist compatible pairs $(A, \mathcal{S})$ such that $R(A)$ is not closed. The last result extends, in the same sense as before, a mixed spline problem, which has at the same time interpolating and smoothing properties. This approach was 
introduced by Bezhaev, Rozhenko and Vasilenko [8], who solved it with hypothesis which are analogous to those of Atteia.

\section{Angles between subspaces}

In what follows, $\mathcal{H}$ and $\mathcal{K}$ are complex Hilbert spaces and $L(\mathcal{H}, \mathcal{K})$ denotes the Banach space of all bounded linear operators from $\mathcal{H}$ into $\mathcal{K}$, with the operator norm. If $\mathcal{H}=\mathcal{K}$ we write $L(\mathcal{H})$. By $L(\mathcal{H})^{+}$we denote the subset of all semidefinite positive operators. Observe that these operators are automatically selfadjoint because we deal with complex Hilbert spaces.

Throughout, $\dot{+}$ denotes a direct sum, $\oplus$ an orthogonal sum and $\ominus$ is the orthogonal substraction, i.e., $\mathcal{M} \ominus \mathcal{N}=\mathcal{M} \cap(\mathcal{N} \cap \mathcal{M})^{\perp}$. Every (bounded linear) projection $Q: \mathcal{H} \rightarrow \mathcal{H}$ produces a (direct sum) decomposition $\mathcal{H}=R(Q) \dot{+} N(Q)$, (where $R$ denotes the range and $N$ is the kernel). The set of all (bounded linear) projections on $\mathcal{H}$ is denoted by $\mathcal{Q}$. Observe that $R(Q)$ is closed, because $R(Q)=N(I-Q)$ and $I-Q$ is also a bounded projection. Conversely, if $\mathcal{H}=\mathcal{M} \dot{+} \mathcal{N}$, where $\mathcal{M}$ and $\mathcal{N}$ are closed subspaces of $\mathcal{H}$, then there exists a unique projection $Q: \mathcal{H} \rightarrow \mathcal{H}$ such that $R(Q)=\mathcal{M}$ and $N(Q)=\mathcal{N}$. Denote by $P_{\mathcal{S} / / \mathcal{T}}$ the projection onto $\mathcal{S}$, with nullspace $\mathcal{T}$, and $P_{\mathcal{S}}=P_{\mathcal{S} / / \mathcal{S}^{\perp}}$, the orthogonal projection onto $\mathcal{S}$. Thus, there is a natural bijection between $\mathcal{Q}$ and the set of all direct sum decompositions $\mathcal{H}=\mathcal{M} \dot{+} \mathcal{N}$. Under this bijection, the set $\mathcal{P}$ of all orthogonal projections on $\mathcal{H}$ corresponds to the set of all orthogonal decompositions $\mathcal{H}=\mathcal{M} \dot{+} \mathcal{M}^{\perp}$

Among the variety of notions of angles between subspaces in a Hilbert space we only need to consider those due to Friedrichs $[\mathbf{2 1}]$ and Dixmier [18]. We follow the excellent survey by Deutsch $[\mathbf{1 6}],[\mathbf{1 7}$, Chapter 9].

Let $\mathcal{S}_{1}$ and $\mathcal{S}_{2}$ be closed subspaces of a Hilbert space $\mathcal{H}$. The angle $\alpha\left(\mathcal{S}_{1}, \mathcal{S}_{2}\right) \in[0, \pi / 2]$ is that whose cosine is

$c\left(\mathcal{S}_{1}, \mathcal{S}_{2}\right)=\sup \left\{|\langle x, y\rangle|: x \in \mathcal{S}_{1} \ominus \mathcal{S}_{2},\|x\| \leq 1, y \in \mathcal{S}_{2} \ominus \mathcal{S}_{1},\|y\| \leq 1\right\}$.

The Dixmier angle $\alpha_{0}\left(\mathcal{S}_{1}, \mathcal{S}_{2}\right) \in[0,2 \pi]$ is that whose cosine is

$$
c_{0}\left(\mathcal{S}_{1}, \mathcal{S}_{2}\right)=\sup \left\{|\langle x, y\rangle|: x \in \mathcal{S}_{1},\|x\| \leq 1, y \in \mathcal{S}_{2},\|y\| \leq 1\right\} .
$$

Both definitions are symmetric in $\mathcal{S}_{1}, \mathcal{S}_{2}$ and they coincide if $\mathcal{S}_{1} \cap \mathcal{S}_{2}=$ $\{0\}$. However, if $\mathcal{S}_{1} \cap \mathcal{S}_{2} \neq\{0\}$ then, automatically, $c_{0}\left(\mathcal{S}_{1}, \mathcal{S}_{2}\right)=1$, but $c\left(\mathcal{S}_{1}, \mathcal{S}_{2}\right)$ depends on the closedness of $\mathcal{S}_{1}+\mathcal{S}_{2}$. More precisely, $c\left(\mathcal{S}_{1}, \mathcal{S}_{2}\right)<1$ if and only if $\mathcal{S}_{1}+\mathcal{S}_{2}$ is closed. By a theorem of Kato [29, Theorem 4.8], it holds that $\mathcal{S}_{1}+\mathcal{S}_{2}$ is closed if and only if $\mathcal{S}_{1}^{\perp}+\mathcal{S}_{2}^{\perp}$ is closed, and in this case $\left(\mathcal{S}_{1} \cap \mathcal{S}_{2}\right)^{\perp}=\mathcal{S}_{1}^{\perp}+\mathcal{S}_{2}^{\perp}$. This also follows 
from the equality $c\left(\mathcal{S}_{1}^{\perp}, \mathcal{S}_{2}^{\perp}\right)=c\left(\mathcal{S}_{1}, \mathcal{S}_{2}\right)$, which is not trivial (see [16, Theorem 16]). For the Dixmier angle, it holds $c_{0}\left(\mathcal{S}_{1}, \mathcal{S}_{2}\right)<1$ if and only if $\mathcal{S}_{1}+\mathcal{S}_{2}$ is closed and $\mathcal{S}_{1} \cap \mathcal{S}_{2}=\{0\}$. In terms of the orthogonal projections $P_{\mathcal{S}_{1}}$ and $P_{\mathcal{S}_{2}}$, the following identities are useful:

$$
c_{0}\left(\mathcal{S}_{1}, \mathcal{S}_{2}\right)=\left\|P_{\mathcal{S}_{1}} P_{\mathcal{S}_{2}}\right\|, \quad c\left(\mathcal{S}_{1}, \mathcal{S}_{2}\right)=\left\|P_{\mathcal{S}_{1}} P_{\mathcal{S}_{2}}-P_{\mathcal{S}_{1} \cap \mathcal{S}_{2}}\right\| .
$$

More than the exact value of $\alpha\left(\mathcal{S}_{1}, \mathcal{S}_{2}\right)$, we are here interested in determining whether $\alpha\left(\mathcal{S}_{1}, \mathcal{S}_{2}\right) \neq 0$, i.e., $c\left(\mathcal{S}_{1}, \mathcal{S}_{2}\right)<1$; analogously for $\alpha_{0}, c_{0}$.

The contents of the next theorem are mostly known, but they are quite sparsed in the literature. Let us mention a paper by Pták [37] for part of item (2), and [13] for item (1). We choose to state and prove it here to emphasize the role of the Dixmier angle in these matters: thus the property "H $\mathcal{H}=\mathcal{S}_{1}+\mathcal{S}_{2}$ " only depends on the angle $\alpha_{0}\left(\mathcal{S}_{1}^{\perp}, \mathcal{S}_{2}^{\perp}\right)$, and the property " $\mathcal{H}=\mathcal{S}_{1} \dot{+} \mathcal{S}_{2}$ " only depends on the angles $\alpha_{0}\left(\mathcal{S}_{1}^{\perp}, \mathcal{S}_{2}^{\perp}\right)$ and $\alpha_{0}\left(\mathcal{S}_{1}, \mathcal{S}_{2}\right)$.

Theorem 2.1. Let $\mathcal{S}_{1}$ and $\mathcal{S}_{2}$ be two closed subspaces of $\mathcal{H}$. Then,

(1) $\mathcal{H}=\mathcal{S}_{1}+\mathcal{S}_{2} \Leftrightarrow c_{0}\left(\mathcal{S}_{1}^{\perp}, \mathcal{S}_{2}^{\perp}\right)<1 \Leftrightarrow\left\|P_{\mathcal{S}_{1}^{\perp}} P_{\mathcal{S}_{2}^{\perp}}\right\|<1$;

(2) $\mathcal{H}=\mathcal{S}_{1} \dot{+} \mathcal{S}_{2} \Leftrightarrow c_{0}\left(\mathcal{S}_{1}, \mathcal{S}_{2}\right)<1$ and $c_{0}\left(\mathcal{S}_{1}^{\perp}, \mathcal{S}_{2}^{\perp}\right)<1$.

Proof: (1) If $\mathcal{H}=\mathcal{S}_{1}+\mathcal{S}_{2}$ then $\mathcal{S}_{1}+\mathcal{S}_{2}$ is obviously closed and, therefore, $c\left(\mathcal{S}_{1}, \mathcal{S}_{2}\right)<1$, which is equivalent to $c\left(\mathcal{S}_{1}^{\perp}, \mathcal{S}_{2}^{\perp}\right)<1$. On the other hand, $\mathcal{S}_{1}^{\perp} \cap \mathcal{S}_{2}^{\perp}=\left(\mathcal{S}_{1}+\mathcal{S}_{2}\right)^{\perp}=\mathcal{H}^{\perp}=\{0\}$. Thus, $c_{0}\left(\mathcal{S}_{1}^{\perp}, \mathcal{S}_{2}^{\perp}\right)<1$, by the comment above. Conversely, if $c_{0}\left(\mathcal{S}_{1}^{\perp}, \mathcal{S}_{2}^{\perp}\right)<1$ then $\mathcal{S}_{1}^{\perp} \cap \mathcal{S}_{2}^{\perp}=\{0\}$ and $\mathcal{S}_{1}^{\perp}+\mathcal{S}_{2}^{\perp}$ is closed. As before, this implies that $\mathcal{S}_{1}+\mathcal{S}_{2}$ is closed; also $\left(\mathcal{S}_{1}+\mathcal{S}_{2}\right)^{\perp}=\mathcal{S}_{1}^{\perp} \cap \mathcal{S}_{2}^{\perp}=\{0\}$. Therefore, $\mathcal{H}=\mathcal{S}_{1}+\mathcal{S}_{2}$. The last equivalence is obvious.

(2) If $\mathcal{H}=\mathcal{S}_{1} \dot{+} \mathcal{S}_{2}$ then $\mathcal{H}=\mathcal{S}_{1}^{\perp} \dot{+} \mathcal{S}_{2}^{\perp}$. Therefore, by the last theorem, we get $c_{0}\left(\mathcal{S}_{1}^{\perp}, \mathcal{S}_{2}^{\perp}\right)<1$ and $c_{0}\left(\mathcal{S}_{1}, \mathcal{S}_{2}\right)<1$. Conversely, if $c_{0}\left(\mathcal{S}_{1}, \mathcal{S}_{2}\right)<1$ then $\mathcal{S}_{1}+\mathcal{S}_{2}$ is closed and $\mathcal{S}_{1} \cap \mathcal{S}_{2}=\{0\}$. But $c_{0}\left(\mathcal{S}_{1}^{\perp}, \mathcal{S}_{2}^{\perp}\right)<1$ implies that $\mathcal{S}_{1}^{\perp} \cap \mathcal{S}_{2}^{\perp}=\{0\}$; since $\mathcal{S}_{1}^{\perp} \cap \mathcal{S}_{2}^{\perp}=\left(\mathcal{S}_{1}+\mathcal{S}_{2}\right)^{\perp}, \mathcal{S}_{1}+\mathcal{S}_{2}$ is dense and, since it is also closed, $\mathcal{S}_{1} \dot{+} \mathcal{S}_{2}=\mathcal{H}$.

This result explains why the property " $c\left(\mathcal{S}_{1}, \mathcal{S}_{2}\right)<1 \Leftrightarrow c\left(\mathcal{S}_{1}^{\perp}, \mathcal{S}_{2}^{\perp}\right)<1$ " does not hold for $c_{0}$, in general. In fact, the equality $\mathcal{H}=\mathcal{S}_{1}+\mathcal{S}_{2}$ is obviously not equivalent to $\mathcal{H}=\mathcal{S}_{1}^{\perp}+\mathcal{S}_{2}^{\perp}$ if the sum $\mathcal{S}_{1}+\mathcal{S}_{2}$ is not direct.

\section{Compatibility}

A positive operator $A \in L(\mathcal{H})^{+}$and a closed subspace $\mathcal{S}$ of $\mathcal{H}$ are called compatible if there exists a bounded linear projection $Q \in L(\mathcal{H})$ with image $\mathcal{S}$ such that $A Q=Q^{*} A$. This means that $Q$ is selfadjoint 
with respect to the semi-inner product $\langle,\rangle_{A}$ given by $\langle x, y\rangle_{A}=\langle A x, y\rangle$. Thus, $Q$ is like an "orthogonal" projection onto $\mathcal{S}$ with respect to $\langle,\rangle_{A}$, or $A$-orthogonal.

Every $Q \in \mathcal{Q}$ is $A$-orthogonal for some $A \in L(\mathcal{H})^{+}$; moreover, $A$ can be chosen to be invertible. For example, if $A=Q^{*} Q+\left(I-Q^{*}\right)(I-Q)$ then $A \in L(\mathcal{H})^{+}$is invertible and $A Q=Q^{*} A$.

Of course, if $A$ is invertible then $\langle,\rangle_{A}$ is an inner product which is equivalent to the original $\langle$,$\rangle and therefore, by the projection the-$ orem, there exists a unique $A$-orthogonal projection over every closed subspace $\mathcal{S}$. Thus, for an invertible $A$, every closed subspace is compatible. However, if $A$ and $\mathcal{S}$ are not compatible, there exists no such projection. Even if $A$ and $\mathcal{S}$ are compatible, there may exist uncountable many such projections. Observe that non compatibility only may occur for infinite dimensional spaces. More precisely, if $\operatorname{dim} A \mathcal{S}<\infty$ then $(A, \mathcal{S})$ is compatible. In fact, if $\operatorname{dim} A \mathcal{S}<\infty$ then $A \mathcal{S}$ is closed and $c_{0}\left(\mathcal{S}^{\perp}, \overline{A \mathcal{S}}\right)=\left\langle s^{\perp}, A s\right\rangle$ for suitable $s^{\perp} \in \mathcal{S}^{\perp}, s \in \mathcal{S}$ with $\left\|s^{\perp}\right\|=$ $\|A s\|=1$. If $c_{0}\left(\mathcal{S}^{\perp}, \overline{A \mathcal{S}}\right)=1$ then Cauchy-Schwarz inequality implies that $s^{\perp}=\lambda A s$ for some scalar $\lambda$ with $|\lambda|=1$, but this is imposible: $A s \in \mathcal{S}^{\perp} \Leftrightarrow A s=0$. On the other side, there are many examples of non compatible pairs. In $[\mathbf{1 3}],[\mathbf{1 4}],[\mathbf{1 5}]$, the reader will find many examples of compatible and non compatible pairs; see also the example at the end of Sard's paper [38].

There are many instances where the original inner product is perturbed or even dramatically changed. The conjugate gradient method of Lanczos, Hestenes and Stiefel (see [31], [26] or [22]) is one of these situations. In general, one needs to change $\langle$,$\rangle by an equivalent inner$ product $\langle,\rangle_{A}$. However, if the invertible operator is what in numerical analysis is called "ill-conditioned" (which, essentially, means that $\left\|A^{-1}\right\|$ is much bigger than $\frac{1}{\|A\|}$ ), then one is forced to consider a positive non invertible $A$. This induces one to study compatibility. The idea is that, in the presence of a suitable compatibility assumption one will get the same type of results as in a finite dimensional setting or in an infinite dimensional one but with a positive invertible $A$.

We present now a short résumé of results on compatibility which contains several theorems proved in $[\mathbf{1 3}],[\mathbf{1 4}],[\mathbf{1 5}]$, together with a set of new results. They are unified by the notions of Dixmier angle and redundant (i.e., non direct) decompositions of the space. In what follows, we first present a characterization of compatibility in terms of Dixmier angles. It is based on the following lemma of M. Krein [30]. 
Lemma 3.1. If $Q \in L(\mathcal{H})$ is a projection with image $\mathcal{S}$ and $A \in L(\mathcal{H})^{+}$, then $A Q=Q^{*} A$ if and only if $N(Q) \subseteq(A \mathcal{S})^{\perp}$.

Proof: If $A Q=Q^{*} A$ and $x \in N(Q)$ then for every $s \in \mathcal{S}$ we get $\langle x, A s\rangle=$ $\left\langle Q^{*} A x, s\right\rangle=\langle A Q x, s\rangle=0$, which proves that $N(Q) \subseteq(A \mathcal{S})^{\perp}$.

Conversely, if $N(Q) \subseteq(A \mathcal{S})^{\perp}$ and $z \in \mathcal{H}$ is decomposed as $z=Q z+$ $(I-Q) z$, for every $x \in \mathcal{H}$ we get $\langle A Q x, z\rangle=\langle A Q x, Q z\rangle=\left\langle Q^{*} A Q x, z\right\rangle$. Thus, $A Q=Q^{*} A Q$, which is Hermitian, so $A Q=Q^{*} A$.

Theorem 3.2. Given a closed subspace $\mathcal{S}$ of $\mathcal{H}$ and $A \in L(\mathcal{H})^{+}$then, the following conditions are equivalent:

(1) $(A, \mathcal{S})$ is compatible;

(2) $\mathcal{H}=\mathcal{S}+(A \mathcal{S})^{\perp}$;

(3) $\left\|P_{\mathcal{S}^{\perp}} P_{\overline{A \mathcal{S}}}\right\|<1$ (or equivalently, $c_{0}\left(\mathcal{S}^{\perp}, \overline{A \mathcal{S}}\right)<1$ ).

The equivalence between (1) and (2) can be found in [13]. However, for the sake of completeness we include a proof.

Proof: $(1) \Leftrightarrow(2)$ : if $(A, \mathcal{S})$ is compatible and $Q$ is a projection over $\mathcal{S}$ such that $A Q=Q^{*} A$, by Krein's lemma $N(Q) \subseteq(A \mathcal{S})^{\perp}$ and therefore $\mathcal{H}=R(Q)+N(Q) \subseteq \mathcal{S}+(A \mathcal{S})^{\perp}$.

Conversely, if $\mathcal{H}=\mathcal{S}+(A \mathcal{S})^{\perp}$ then $\mathcal{H}=\mathcal{S} \dot{+}(A \mathcal{S})^{\perp} \ominus \mathcal{S}$. Then, by Krein's lemma, again, the projection onto $\mathcal{S}$ defined by the last decomposition satisfies $A Q=Q^{*} A$, which shows that $(A, \mathcal{S})$ is compatible.

The equivalence between (2) and (3) is a rewriting of Theorem 2.1.

Remark 3.3. Given $A, B \in L(\mathcal{H})^{+}$and a closed subspace $\mathcal{S}$ such that $\overline{A \mathcal{S}}=\overline{B \mathcal{S}}$ then $(A, \mathcal{S})$ is compatible if and only if $(B, \mathcal{S})$ is compatible.

In $[\mathbf{1 5}$, Proposition 2.14] it was proven that the compatibility between $A \in L(\mathcal{H})^{+}$and a subspace $\mathcal{S}$ is equivalent to the direct decomposition $R(A)=A \mathcal{S} \dot{+} R(A) \cap \mathcal{S}^{\perp}$. For a later use see Theorem 4.6. We extend this result to non necessarily positive operators. More precisely:

Proposition 3.4. Let $T \in L(\mathcal{H})$ and let $\mathcal{S}$ be a closed subspace of $\mathcal{H}$. If $A=T^{*} T$ then the following conditions are equivalent:

(1) the pair $(A, \mathcal{S})$ is compatible;

(2) $R(T)=T \mathcal{S} \oplus(T \mathcal{S})^{\perp} \cap R(T)$;

(3) if $\mathcal{M}=\overline{T \mathcal{S}}$, then $R\left(P_{\mathcal{M}} T\right) \subseteq T \mathcal{S}$.

Proof: We use the fact that $(A \mathcal{S})^{\perp}=A^{-1}\left(\mathcal{S}^{\perp}\right)$.

$(1) \Leftrightarrow(2):$ If $(A, \mathcal{S})$ is compatible then, by Theorem $3.2, \mathcal{H}=\mathcal{S}+(A \mathcal{S})^{\perp}$ and applying $T$ to both sides of the equality we get that $R(T)=T \mathcal{S}+$ 
$T A^{-1}\left(\mathcal{S}^{\perp}\right)$. Observe that

$T A^{-1}\left(\mathcal{S}^{\perp}\right)=T\left(T^{*} T\right)^{-1}\left(\mathcal{S}^{\perp}\right)=T T^{-1}\left(T^{*-1}\left(\mathcal{S}^{\perp}\right)\right)=T^{*-1}\left(\mathcal{S}^{\perp}\right) \cap R(T)$.

But $T^{*-1}\left(\mathcal{S}^{\perp}\right)=(T \mathcal{S})^{\perp}$ so that $T A^{-1}\left(\mathcal{S}^{\perp}\right)=(T \mathcal{S})^{\perp} \cap R(T)$. Therefore $R(T)=T \mathcal{S} \oplus(T \mathcal{S})^{\perp} \cap R(T)$. The converse is similar.

(2) $\Leftrightarrow(3)$ : If $y \in R(T)$ then $y=y_{1}+y_{2}$ for unique $y_{1} \in T \mathcal{S}$ and $y_{2} \in(T \mathcal{S})^{\perp}$, then $P_{\mathcal{M}} y=y_{1} \in T \mathcal{S}$. The converse is similar.

Corollary 3.5. Let $\mathcal{S}$ be a closed subspace of $\mathcal{H}$ and $A \in L(\mathcal{H})^{+}$with closed range, then $(A, \mathcal{S})$ is compatible if and only if $\mathcal{S}+N(A)$ is closed, or equivalently, if $A \mathcal{S}$ is closed.

Proof: It is easy to see that if $A$ has closed range then $\mathcal{S}+N(A)$ is closed if and only if $A \mathcal{S}$ is closed: in fact, since $R(A)$ is closed then $A: \mathcal{H} \rightarrow$ $R(A)$ is a quotion map, by the Open Mapping theorem. Therefore $A \mathcal{S}$ is closed if and only if $\mathcal{S}+N(A)$ is closed.

Observe that $R(A)=R\left(A^{1 / 2}\right)$ because $R(A)$ is closed. If $(A, \mathcal{S})$ is compatible, applying Proposition 3.4, with $T=A^{1 / 2}$, we get that $R\left(A^{1 / 2}\right)=A^{1 / 2} \mathcal{S} \oplus\left(A^{1 / 2} \mathcal{S}\right)^{\perp} \cap R\left(A^{1 / 2}\right)$. Then, the set $A^{1 / 2} \mathcal{S}$ must be closed because the decomposition of $R\left(A^{1 / 2}\right)$ is orthogonal and $R\left(A^{1 / 2}\right)$ is closed. It follows that $\mathcal{S}+N\left(A^{1 / 2}\right)=\mathcal{S}+N(A)$ is closed. Conversely, suppose that $\mathcal{S}+N(A)$ is closed, then $A^{1 / 2} \mathcal{S}$ is closed. In this case, it is easy to see that $R\left(A^{1 / 2}\right)=A^{1 / 2} \mathcal{S} \oplus\left(A^{1 / 2} \mathcal{S}\right)^{\perp} \cap R\left(A^{1 / 2}\right)$, so that $(A, \mathcal{S})$ is compatible, by Proposition 3.4.

Corollary 3.6. Given closed subspaces $\mathcal{M}, \mathcal{S}$ such that $\mathcal{S}^{\perp} \cap \mathcal{M}=\{0\}$, the pair $\left(P_{\mathcal{M}}, \mathcal{S}\right)$ is compatible if and only if $\left\|P_{\mathcal{S}^{\perp}} P_{\mathcal{M}}\right\|<1$.

Proof: By Corollary 3.5, the pair $\left(P_{\mathcal{M}}, \mathcal{S}\right)$ is compatible if and only if $\mathcal{S}+\mathcal{M}^{\perp}$ is closed, or equivalently, if $\mathcal{S}^{\perp}+\mathcal{M}$ is closed, or $c\left(\mathcal{S}^{\perp}, \mathcal{M}\right)<$ 1. By $(1), c\left(\mathcal{S}^{\perp}, \mathcal{M}\right)=\left\|P_{\mathcal{S}^{\perp}} P_{\mathcal{M}}-P_{\mathcal{S}^{\perp} \cap \mathcal{M}}\right\|=\left\|P_{\mathcal{S}^{\perp}} P_{\mathcal{M}}\right\|$, because $\mathcal{S}^{\perp} \cap \mathcal{M}=\{0\}$.

Example 3.7. The following example is due to J. Antezana. Let $\mathbb{T}=$ $\{z \in \mathbb{C}:|z|=1\}$. Consider the space $L^{2}(\mathbb{T})$ and its subspaces $H^{2}(\mathbb{T})$, $\mathcal{S}=\left\{f \in L^{2}(\mathbb{T}): f(z)=0\right.$ if $\left.\operatorname{Im} z \geq 0\right\}$ and $\mathcal{S}^{\perp}=\left\{f \in L^{2}(\mathbb{T})\right.$ : $f(z)=0$ if $\operatorname{Im} z \leq 0\}$. Every $\psi \in L^{\infty}(\mathbb{T})$ defines a multiplication operator $M_{\psi}: f \rightarrow \psi f$ in $L\left(L^{2}(\mathbb{T})\right)$ and a Toeplitz operator $T_{\psi}=P M_{\psi} P$ in $L\left(L^{2}(\mathbb{T})\right.$ ), where $P$ is the orthogonal projection onto $H^{2}(\mathbb{T})$ (the socalled Riesz projection). Observe that $P_{\mathcal{S}}=M_{\chi}$, where $\chi(z)=0$ if $\operatorname{Im} z \leq 0, \chi(z)=1$ if $\operatorname{Im} z \geq 0$, analogously $P_{\mathcal{S}^{\perp}}=M_{I-\chi}$. It is well known that $\left\|T_{\psi}\right\|=\|\psi\|_{\infty}$ and $H^{2}(\mathbb{T}) \cap \mathcal{S}=H^{2}(\mathbb{T}) \cap \mathcal{S}^{\perp}=\{0\}$ (see, for instance, [34]). 
It holds $\left\|P P_{\mathcal{S}^{\perp}}\right\|=1$ : in fact, $1 \geq\left\|P P_{\mathcal{S}^{\perp}}\right\|=\left\|P M_{1-\chi}\right\| \geq\left\|P M_{I-\chi} P\right\|=$ $\left\|T_{I-\chi}\right\|=\|I-\chi\|_{\infty}=1$. This implies, by Corollary 3.6, that the pair $(P, \mathcal{S})$ is not compatible.

Suppose that $\mathcal{M}$ and $\mathcal{S}$ are two closed subspaces of $\mathcal{H}$ such that $\mathcal{H}=$ $\mathcal{S}+\mathcal{M}^{\perp}$ and consider the set

$$
\mathcal{P}(\mathcal{S}, \mathcal{M})=\left\{Q \in \mathcal{Q}: R(Q)=\mathcal{S}, N(Q) \subseteq \mathcal{M}^{\perp}\right\} .
$$

The next theorem explicity shows that $\mathcal{P}(\mathcal{S}, \mathcal{M})$ is an affine submanifold of $L(\mathcal{H})$. It will be useful in the study of compatibility.

Theorem 3.8. Let $\mathcal{M}$ and $\mathcal{S}$ be two closed subspaces of $\mathcal{H}$ such that $\mathcal{H}=\mathcal{S}+\mathcal{M}^{\perp}$, then

$\mathcal{P}(\mathcal{S}, \mathcal{M})=P_{\mathcal{S} / / \mathcal{M}^{\perp} \ominus \mathcal{S}}+\left\{W \in L(\mathcal{H}): R(W) \subseteq \mathcal{S} \cap \mathcal{M}^{\perp}\right.$ and $\left.\mathcal{S} \subseteq N(W)\right\}$.

Proof: Let $\mathcal{N}=\mathcal{S} \cap \mathcal{M}^{\perp}$. If $Q \in \mathcal{P}(\mathcal{S}, \mathcal{M})$, consider $W=Q-P_{\mathcal{S} / / \mathcal{M}^{\perp} \ominus \mathcal{S}}$. Then, $Q=P_{\mathcal{S} / / \mathcal{M}^{\perp} \ominus \mathcal{S}}+W, R(W) \subseteq \mathcal{S}$ and also $R(W) \subseteq \mathcal{M}^{\perp}$, because we can write $W=\left(I-P_{\mathcal{S} / / \mathcal{M}^{\perp} \ominus \mathcal{S}}\right)-(I-Q)$. Therefore $R(W) \subseteq \mathcal{N}$. It is obvious that $\mathcal{S} \subseteq N(W)$.

Conversely, if $Q=P_{\mathcal{S} / / \mathcal{M}^{\perp} \ominus \mathcal{S}}+W$, where $R(W) \subseteq \mathcal{N}$ and $\mathcal{S} \subseteq N(W)$, it follows that $P_{\mathcal{S} / / \mathcal{M}^{\perp} \ominus \mathcal{S}} W=W, W P_{\mathcal{S} / / \mathcal{M}^{\perp} \ominus \mathcal{S}}=0$ and $W^{2}=0$; therefore $Q^{2}=Q$. Also $Q s=s$, for all $s \in \mathcal{S}$ and $R(Q) \subseteq \mathcal{S}$, so that $R(Q)=\mathcal{S}$; finally, if $Q x=0$ then $P_{\mathcal{S} / / \mathcal{M}^{\perp} \ominus \mathcal{S}} x=-W x \in \mathcal{N}$ so that $x=P_{\mathcal{S} / / \mathcal{M}^{\perp} \ominus \mathcal{S}} x+\left(I-P_{\mathcal{S} / / \mathcal{M}^{\perp} \ominus \mathcal{S}}\right) x \in \mathcal{M}^{\perp}$. Then $Q \in \mathcal{P}(\mathcal{S}, \mathcal{M})$.

The next one is a kind of dual of the theorem above.

Corollary 3.9. Suppose that $\mathcal{M}$ and $\mathcal{S}$ are two closed subspaces of $\mathcal{H}$ such that $\mathcal{M}+\mathcal{S}^{\perp}$ is a proper closed subspace of $\mathcal{H}$ and $\mathcal{M} \cap \mathcal{S}^{\perp}=\{0\}$. Consider the set

$$
\mathcal{P}^{*}(\mathcal{S}, \mathcal{M})=\left\{Q \in \mathcal{Q}: N(Q)=\mathcal{S}^{\perp}, \mathcal{M} \subseteq R(Q)\right\} .
$$

Then, $\mathcal{P}^{*}(\mathcal{S}, \mathcal{M})=P_{\mathcal{M}+\mathcal{S} \cap \mathcal{M}^{\perp} / / \mathcal{S}^{\perp}}+\left\{W \in L(\mathcal{H}), R(W) \subseteq \mathcal{S}^{\perp}, \mathcal{M}+\right.$ $\left.\mathcal{S}^{\perp} \subseteq N(W)\right\}$.

Proof: It suffices to notice that $\mathcal{P}^{*}(\mathcal{S}, \mathcal{M})=\mathcal{P}(\mathcal{S}, \mathcal{M})^{*}$ and that if $\mathcal{M}+$ $\mathcal{S}^{\perp}$ is closed then $\left(\mathcal{S}+\mathcal{M}^{\perp}\right)^{\perp}=\overline{\mathcal{M}+\mathcal{S}^{\perp}}=\mathcal{M}+\mathcal{S}^{\perp}$.

In Section 2, devoted to angles between subspaces, we proved that two subspaces span the whole space if and only if its Dixmier angle is not 0 . We prove now a different characterization, this time in terms of the notion of compatibility. 
Theorem 3.10. Let $\mathcal{S}, \mathcal{M}$ be closed subspaces of $\mathcal{H}$. Then the following conditions are equivalent:

(1) $\mathcal{H}=\mathcal{S}+\mathcal{M}^{\perp}$

(2) there exists a projection $Q \in L(\mathcal{H})$ with image $\mathcal{S}$ such that $P_{\mathcal{M}} Q=$ $P_{\mathcal{M}}$

(3) $P_{\mathcal{M}} \mathcal{S}=\mathcal{M}$ and the pair $\left(P_{\mathcal{M}}, \mathcal{S}\right)$ is compatible;

(4) there exists $A \in L(\mathcal{H})^{+}$such that $A \mathcal{S}=\mathcal{M}$ and the pair $(A, \mathcal{S})$ is compatible.

Proof: $(1) \Rightarrow(2)$ : Even if $\mathcal{H}=\mathcal{S}+\mathcal{M}^{\perp}$ is a non direct sum, the decomposition $\mathcal{H}=\mathcal{S}+\mathcal{M}^{\perp} \ominus \mathcal{S}$ is direct. Let $Q \in L(\mathcal{H})$ be the projection with image $R(Q)=\mathcal{S}$ and nullspace $N(Q)=\mathcal{M}^{\perp} \ominus \mathcal{S} \subseteq \mathcal{M}^{\perp}$. Therefore, $P_{\mathcal{M}}(I-Q)=0$ so that $P_{\mathcal{M}}=P_{\mathcal{M}} Q$.

$(2) \Rightarrow(3)$ : Let $Q \in L(\mathcal{H})$ be the projection with image $R(Q)=\mathcal{S}$ and $P_{\mathcal{M}}=P_{\mathcal{M}} Q$ then $P_{\mathcal{M}} Q=Q^{*} P_{\mathcal{M}}$ and $\left(P_{\mathcal{M}}, \mathcal{S}\right)$ is compatible. On the other hand, $P_{\mathcal{M}} \mathcal{S}=R\left(P_{\mathcal{M}} Q\right)=R\left(P_{\mathcal{M}}\right)=\mathcal{M}$.

(3) $\Rightarrow$ (4): Take $A=P_{\mathcal{M}}$.

(4) $\Rightarrow(1)$ : If $(A, \mathcal{S})$ is compatible then $\mathcal{H}=\mathcal{S}+(A \mathcal{S})^{\perp}$. Since $A \mathcal{S}=\mathcal{M}$, we get $\mathcal{H}=\mathcal{S}+\mathcal{M}^{\perp}$.

Remark 3.11. 1. The choice of $\mathcal{M}^{\perp}$ instead of $\mathcal{M}$ in the definition of $\mathcal{P}(\mathcal{S}, \mathcal{M})$ and in condition (1), simplifies the form of the other conditions (2), (3) and (4).

2. If $\mathcal{H}$ is infinite-dimensional and $\mathcal{S}$ is finite-dimensional, then $\mathcal{M}$ is also finite-dimensional.

3. A consequence of the theorem is that one is forced to admit non invertible positive operators when dealing with non-direct sums.

\section{Consistent reconstruction of signals.}

The notion of consistent reconstruction in signal processing was introduced by Unser and Aldroubi [40]. Let $\mathcal{H}$ be a Hilbert space of functions ("signals") and consider two closed subspaces of $\mathcal{H}$ : the sampling space $\mathcal{V}_{s}$ and the reconstruction space $\mathcal{V}_{r}$. Suppose that for $h \in \mathcal{H}$, the scalars

$$
c_{i}=\left\langle h, s_{i}\right\rangle, \quad i \in I
$$

are known, for a fixed set of "sampling vectors" $\left\{s_{i}\right\}_{i \in I}$ which spans $\mathcal{V}_{s}$. A process of linear reconstruction of signals consists of a linear operator which assigns to any $h \in \mathcal{H}$ a certain $\tilde{h} \in \mathcal{V}_{r}$, satisfying that if $h \in \mathcal{V}_{r}$ then $\tilde{h}=h$ and if $P_{\mathcal{V}_{s}} \tilde{h}=0$ then $\tilde{h}=0$ (uniqueness condition) and 
$P_{\mathcal{V}_{s}} \tilde{h}=P_{\mathcal{V}_{s}} h$, for $h \in \mathcal{H}$ (consistency condition). It is easy to see that the first condition implies that $\mathcal{V}_{r} \cap \mathcal{V}_{s}^{\perp}=\{0\}$ : suppose that $h \in \mathcal{V}_{r} \cap \mathcal{V}_{s}^{\perp}$; then, by the first condition, $\tilde{h}=h$ and $P_{\mathcal{V}_{s}} \tilde{h}=0$, because $h \in \mathcal{V}_{s}^{\perp}$; therefore $\tilde{h}=0$, so that $h=0$.

In [20] Eldar proved that if $\mathcal{V}_{r} \dot{+} \mathcal{V}_{s}^{\perp}=\mathcal{H}$, there exists a unique consistent reconstruction of $h$ given by $\tilde{h}=P_{\mathcal{V}_{r} / / \mathcal{V}_{s}^{\perp}} h$. The following corollary of Theorem 3.10 establishes a relation between the notion of compatibility and the existence of consistent reconstructions.

Corollary 3.12. Given $\mathcal{V}_{s}$ and $\mathcal{V}_{r}$ two closed subspaces of $\mathcal{H}$, there exists a consistent reconstruction with sampling space $\mathcal{V}_{s}$ and reconstruction space $\mathcal{V}_{r}$ if and only if the pair $\left(P_{\mathcal{V}_{s}}, \mathcal{V}_{r}\right)$ is compatible and $\mathcal{V}_{r} \cap \mathcal{V}_{s}^{\perp}=\{0\}$.

Even if $\mathcal{H}=\mathcal{V}_{r}+\mathcal{V}_{s}^{\perp}$ but the sum is not direct, one can always construct a consistent reconstruction $Q$ with a smaller reconstruction space $R(Q) \subseteq \mathcal{V}_{r}$. In fact, if we choose any subspace $\tilde{\mathcal{V}}_{r} \subseteq \mathcal{V}_{r}$ such that $\mathcal{H}=\tilde{\mathcal{V}}_{r} \dot{+} \mathcal{V}_{s}^{\perp}$ then $Q=P_{\tilde{\mathcal{V}}_{r} / / \mathcal{V}_{s}^{\perp}}$ is the unique consistent reconstruction with sampling space $\mathcal{V}_{s}$ and reconstruction space $\tilde{\mathcal{V}}_{r}[\mathbf{2 7}]$.

\section{The set $\mathcal{P}(A, \mathcal{S})$}

We fix some notations. If $(A, \mathcal{S})$ is a compatible pair, $\mathcal{P}(A, \mathcal{S})$ denotes the set of all $A$-orthogonal projections onto $\mathcal{S}$. Denote by $\mathcal{N}=\mathcal{S} \cap(A \mathcal{S})^{\perp}$; it is easy to see that $\mathcal{N}=\mathcal{S} \cap N(A)$.

Lemma 4.1. If $\mathcal{H}=\mathcal{S}_{1}+\mathcal{S}_{2}$ for some closed subspaces $\mathcal{S}_{1}, \mathcal{S}_{2}$ of $\mathcal{H}$ then the set of closed complements of $\mathcal{S}_{1} \cap \mathcal{S}_{2}$ in $\mathcal{S}_{2}$ coincides with the set of closed complements of $\mathcal{S}_{1}$ which are contained in $\mathcal{S}_{2}$, i.e.

$$
\begin{aligned}
\mathcal{A}_{\mathcal{S}_{1}, \mathcal{S}_{2}} & =\left\{\mathcal{M} \in \operatorname{Gr}(\mathcal{H}): \mathcal{H}=\mathcal{S}_{1} \dot{+} \mathcal{M}, \mathcal{M} \subseteq \mathcal{S}_{2}\right\} \\
& =\left\{\mathcal{M} \in \operatorname{Gr}(\mathcal{H}): \mathcal{S}_{2}=\mathcal{M} \dot{+} \mathcal{S}_{1} \cap \mathcal{S}_{2}\right\} .
\end{aligned}
$$

Proof: Straightforward.

Theorem 4.2. Let $(A, \mathcal{S})$ be a compatible pair in $\mathcal{H}$. Then

$$
\mathcal{P}(A, \mathcal{S})=\left\{Q \in \mathcal{Q}: R(Q)=\mathcal{S}, N(Q) \in \mathcal{A}_{\mathcal{S},(A \mathcal{S})^{\perp}}\right\} .
$$

Proof: By Krein's lemma, if $Q \in \mathcal{Q}$ and $R(Q)=\mathcal{S}$ then $Q \in \mathcal{P}(A, \mathcal{S})$ if and only if $N(Q) \subseteq(A \mathcal{S})^{\perp}$. If we identify each $Q \in \mathcal{Q}$ with the decomposition $\mathcal{H}=R(Q) \dot{+} N(Q)$, the theorem follows from the lemma above.

Observe that, if $\mathcal{H}=\mathcal{S}_{1}+\mathcal{S}_{2}$ as before there is a distinguished element of $\mathcal{A}_{\mathcal{S}_{1}, \mathcal{S}_{2}}$, namely, $\mathcal{M}=\mathcal{S}_{2} \ominus \mathcal{S}_{1}$. In particular, we get a distinguished projection in $\mathcal{P}(A, \mathcal{S})$. More precisely, $P_{A, \mathcal{S}} \in \mathcal{P}(A, \mathcal{S})$ is the unique 
projection such that $R\left(P_{A, \mathcal{S}}\right)=\mathcal{S}$ and $N\left(P_{A, \mathcal{S}}\right)=(A \mathcal{S})^{\perp} \ominus \mathcal{N}$. Notice that $P_{A, \mathcal{S}}$ implicitly appeared in the proof of Theorem 3.2.

Now, we use $P_{A, \mathcal{S}}$ to characterize the set $\mathcal{P}(A, \mathcal{S})$. An analogous description of this set appears in [13]. We include a one-line proof.

Proposition 4.3. Let $(A, \mathcal{S})$ be a compatible pair. Then

$$
\mathcal{P}(A, \mathcal{S})=P_{A, \mathcal{S}}+\{W \in L(\mathcal{H}): R(W) \subseteq \mathcal{N} \text { and } \mathcal{S} \subseteq N(W)\} .
$$

In particular, if $\mathcal{N}=\{0\}$ then $\mathcal{P}(A, \mathcal{S})=\left\{P_{A, \mathcal{S}}\right\}$.

Proof: Notice that $\mathcal{P}(A, \mathcal{S})=\mathcal{P}(\mathcal{S}, \mathcal{M})$, with $\mathcal{M}=\overline{A \mathcal{S}}$ and apply Theorem 3.8 .

Remark 4.4. Observe that Theorem 3.10 proves that if $\mathcal{H}=\mathcal{S}+\mathcal{M}^{\perp}$ then there exists a positive operator $A \in L(\mathcal{H})$ such that $\mathcal{P}(\mathcal{S}, \mathcal{M})=\mathcal{P}(A, \mathcal{S})$; in fact, if $A=P_{\mathcal{M}}$ then $(A, \mathcal{S})$ is compatible and $\mathcal{P}(\mathcal{S}, \mathcal{M})=\mathcal{P}(A, \mathcal{S})$.

The next result offers an easy expression for $P_{A, \mathcal{S}}$. For its proof we need the following theorem by R. G. Douglas $[\mathbf{1 9}]$ :

Theorem 4.5. Let $A, B \in L(\mathcal{H})$. Then the following conditions are equivalent:

(1) $R(B) \subseteq R(A)$;

(2) there exists $D \in L(\mathcal{H})$ such that $B=A D$.

Moreover, in this case there exists a unique solution $D$ of the equation $A X=B$ such that $R(D) \subseteq \overline{R\left(A^{*}\right)}$ (the Douglas solution) and it holds $\|D\|^{2}=\inf \left\{\lambda: B B^{*} \leq \lambda A A^{*}\right\}$.

Theorem 4.6. Consider $T \in L(\mathcal{H})$ and $\mathcal{S}$ a closed subspace of $\mathcal{H}$. Let $A=T^{*} T, P=P_{\mathcal{S}}$ and suppose that the pair $(A, \mathcal{S})$ is compatible. Then,

$$
P_{A, \mathcal{S} \ominus \mathcal{N}}=(T P)^{\dagger} T=\left(A^{1 / 2} P\right)^{\dagger} A^{1 / 2} .
$$

In particular, if $\mathcal{N}=\{0\}$, it holds $P_{A, \mathcal{S}}=(T P)^{\dagger} T=\left(A^{1 / 2} P\right)^{\dagger} A^{1 / 2}$.

Proof: Suppose that $(A, \mathcal{S})$ is compatible and consider $\mathcal{M}=\overline{T \mathcal{S}}$. By item (3) of Proposition 3.4 and Theorem 4.5, equation $T P X=P_{\mathcal{M}} T$ admits a solution. Let $D$ be the Douglas solution. Then, by $[4], D=$ $(T P)^{\dagger} P_{\mathcal{M}} T$ (notice that $(T P)^{\dagger}$ may be unbounded, but the product $(T P)^{\dagger} P_{\mathcal{M}} T$ is bounded). Observe that $\overline{R(T P)}=\overline{T \mathcal{S}}=\mathcal{M}$, so that $\mathcal{M}^{\perp}=R(T P)^{\perp}=N\left((T P)^{\dagger}\right)$. Therefore $(T P)^{\dagger}\left(I-P_{\mathcal{M}}\right)=0$ or, equivalently, $(T P)^{\dagger}=(T P)^{\dagger} P_{\mathcal{M}}$. This equality shows that $D=(T P)^{\dagger} T$. 
In what follows we prove that $(T P)^{\dagger} T=P_{A, \mathcal{S} \ominus \mathcal{N}}$ : first notice that $R(D) \subseteq \mathcal{S} \ominus \mathcal{N}$ : in fact, $N(T P)=\mathcal{N} \oplus \mathcal{S}^{\perp}$, because $T P h=0$, for $h \in \mathcal{H}$, if and only if $P h \in N(T) \cap \mathcal{S}=\mathcal{N}$, or equivalently, $h \in$ $P^{-1}(\mathcal{N})=\mathcal{N} \oplus \mathcal{S}^{\perp}$. Then $R\left((T P)^{\dagger}\right)=N(T P)^{\perp}=\left(\mathcal{N} \oplus \mathcal{S}^{\perp}\right)^{\perp}=$ $\mathcal{S} \cap \mathcal{N}^{\perp}=\mathcal{S} \ominus \mathcal{N}$. Also $N(D)=(A \mathcal{S})^{\perp}: D x=0$ if and only if $T x \in N\left((T P)^{\dagger}\right)=(T \mathcal{S})^{\perp}=T^{*-1}\left(\mathcal{S}^{\perp}\right)$. Then $x \in N(D)$ if and only if $x \in T^{-1}\left(T^{*-1}\left(\mathcal{S}^{\perp}\right)\right)=A^{-1}\left(\mathcal{S}^{\perp}\right)=(A \mathcal{S})^{\perp}$.

Finally, since $D=P D$, we get $D^{2}=D P D=(T P)^{\dagger} T P D=P_{N(T P)^{\perp}} D=$ $P_{\mathcal{S} \ominus \mathcal{N}} D=D$ (here we use the general fact that even if an operator $B$ does not have closed range, one can define its Moore-Penrose inverse $B^{\dagger}$, which is unbounded; in this case $B^{\dagger} B$ is bounded and satisfies $B^{\dagger} B=P_{N(B)^{\perp}}$, see $\left.[\mathbf{3 3}]\right)$. Therefore, $D$ is a projection, with $R(D) \subseteq$ $\mathcal{S} \ominus \mathcal{N}$ and $N(D)=(A \mathcal{S})^{\perp}$. To see that $R(D)=\mathcal{S} \ominus \mathcal{N}$, observe that $\mathcal{H}=(\mathcal{S} \ominus \mathcal{N}) \dot{+}(A \mathcal{S})^{\perp}$ because $(A, \mathcal{S})$ is compatible, then if $s \in \mathcal{S} \ominus \mathcal{N}$, write $s=D s+(I-D) s$, so that $(I-D) s \in(\mathcal{S} \ominus \mathcal{N}) \cap(A \mathcal{S})^{\perp}=\{0\}$ and $s=D s \in R(D)$; thus $D=P_{A, \mathcal{S} \ominus \mathcal{N}}$.

To prove the second equality, consider $\mathcal{M}^{\prime}=\overline{A^{1 / 2} \mathcal{S}}$ instead of $\mathcal{M}$. Applying [15, Proposition 2.14], equation $A^{1 / 2} P X=P_{\mathcal{M}^{\prime}} A^{1 / 2}$ admits a solution. Let $D^{\prime}$ be the Douglas solution. Following the same steps as before, it can be proved that $D^{\prime}=P_{A, \mathcal{S} \ominus \mathcal{N}}$.

\section{Restricted pseudoinverses.}

Consider $T \in L\left(\mathcal{H}_{1}, \mathcal{H}_{2}\right)$ and $S \in L\left(\mathcal{H}_{1}, \mathcal{H}_{3}\right)$, with $\mathcal{S}=N(S)$. In [32] Minamide and Nakamura defined the pseudoinverse of $T$ restricted to $\mathcal{S}$, denoted by $T_{\mathcal{S}}^{\dagger}$, as the Moore-Penrose inverse of $T_{\mid N(\mathcal{S})}$, provided that $T N(S)$ is closed. They proved that $T_{\mathcal{S}}^{\dagger}$ is the unique solution of the equations

$$
\begin{gathered}
S X=0, \quad X T X=X, \quad(T X)^{*}=T X, \\
T X T=T \text { on } \mathcal{S}, \quad P_{\mathcal{S}}(X T)^{*}=X T \text { on } \mathcal{S} .
\end{gathered}
$$

In the same way as the ordinary pseudoinverse, the restricted pseudoinverse provides the best approximation, when certain constrained problems are considered. It is not difficult to see that $T_{\mathcal{S}}^{\dagger}=\left(T P_{\mathcal{S}}\right)^{\dagger}$; see $[\mathbf{3 2}]$ and $[\mathbf{7}$, p. 91$]$.

Observe that, in general, $T P_{\mathcal{S}}$ is not a closed range operator, so that $T_{\mathcal{S}}^{\dagger}=\left(T P_{\mathcal{S}}\right)^{\dagger}$ is an unbounded densely defined operator; by this reason, Minamide and Nakamura added the hypothesis of the closedness of $T \mathcal{S}=$ $R\left(T P_{\mathcal{S}}\right)$. However, in the presence of a compability hypothesis, the operator $(T S)^{\dagger} T$ is a bounded projection even if $R(T \mathcal{S})$ is not closed. More precisely: 
Corollary 4.7. If $\left(T^{*} T, \mathcal{S}\right)$ is compatible, then

$$
P_{A, \mathcal{S} \ominus \mathcal{N}}=\left(T_{\mathcal{S}}\right)^{\dagger} T=\left(A_{\mathcal{S}}^{1 / 2}\right)^{\dagger} A^{1 / 2} .
$$

In particular, if $\mathcal{N}=\{0\}$, it holds $P_{A, \mathcal{S}}=\left(T_{\mathcal{S}}\right)^{\dagger} T=\left(A_{\mathcal{S}}^{1 / 2}\right)^{\dagger} A^{1 / 2}$.

Proof: It is a rewriting of the previous proposition.

\section{Variational properties of $\boldsymbol{P}_{A, \mathcal{S}}$}

In this section, the projections of the set $\mathcal{P}(A, \mathcal{S})$ and the distinguished projection $P_{A, \mathcal{S}}$ are characterized as solutions of different variational problems.

Given $\mathcal{S}$ a closed subspace of $\mathcal{H}$ the orthogonal projection onto $\mathcal{S}^{\perp}$ is the unique solution to the variational problem

$$
\min _{Q \in \mathcal{Q}, N(Q)=\mathcal{S}} Q^{*} Q
$$

To prove this assertion observe that if $Q \in \mathcal{Q}$ satisfies that $N(Q)=\mathcal{S}$ then the matrix representation of $Q$, in terms of the decomposition $\mathcal{H}=$ $\mathcal{S} \oplus \mathcal{S}^{\perp}$, is $Q=\left(\begin{array}{ll}0 & x \\ 0 & 1\end{array}\right)$, where $x \in L\left(\mathcal{S}^{\perp}, \mathcal{S}\right)$ and 1 is the identity operator of $\mathcal{S}^{\perp}$. Then $Q^{*} Q=\left(\begin{array}{cc}0 & 0 \\ 0 & 1+x^{*} x\end{array}\right)$ and therefore, $Q^{*} Q-P_{\mathcal{S}^{\perp}}=\left(\begin{array}{cc}0 & 0 \\ 0 & x^{*} x\end{array}\right) \geq 0$.

Suppose that $Q_{0} \in \mathcal{Q}$, with $N\left(Q_{0}\right)=\mathcal{S}$ is another solution of the variational problem. Then, $Q_{0}^{*} Q_{0}=P_{\mathcal{S}^{\perp}}$, so that $0=Q_{0}^{*} Q_{0}-P_{\mathcal{S}^{\perp}}=$ $\left(\begin{array}{cc}c c 0 & 0 \\ 0 & x^{*} x\end{array}\right)$; therefore $x^{*} x=0$ and $x=0$.

The symmetric problem

$$
\min _{Q \in \mathcal{Q}, R(Q)=\mathcal{S}} Q Q^{*}
$$

has a unique solution given again by the orthogonal projection $P_{\mathcal{S}}$.

These results are interesting because they prove that $P_{\mathcal{S}}$ is optimal (in a precise sense) among all the projections with image $\mathcal{S}$. We prove now that $P_{A, \mathcal{S}}$ is optimal, in a similar sense, among the projections in $\mathcal{P}(A, \mathcal{S})$. Observe that, even if $P_{A, \mathcal{S}}$ has optimal operator norm in $\mathcal{P}(A, \mathcal{S})$, it is not the unique projection in $\mathcal{P}(A, \mathcal{S})$ with this property (see [13, Theorem 3.5.5]).

Theorem 5.1 ([14, Theorem 3.2.4]). Let $\mathcal{S}$ be a closed subspace of $\mathcal{H}$ and $A \in L(\mathcal{H})^{+}$. If the pair $(A, \mathcal{S})$ is compatible then for every $Q \in$ $\mathcal{P}(A, \mathcal{S})$ and every $h \in \mathcal{H}$

$$
\left\|\left(I-P_{A, \mathcal{S}}\right) h\right\| \leq\|(I-Q) h\| .
$$

Moreover, $\left(I-P_{A, \mathcal{S}}\right) h$ is the unique vector in the set $\{(I-Q) h: Q \in$ $\mathcal{P}(A, \mathcal{S})\}$ with minimal norm. 
Proposition 5.2. Let $\mathcal{S}$ and $\mathcal{M}$ closed subspaces of $\mathcal{H}$ such that $\mathcal{S}+$ $\mathcal{M}^{\perp}=\mathcal{H}$ and consider the set $\mathcal{A}=\left\{Q \in \mathcal{Q}: R(Q) \subseteq \mathcal{M}^{\perp}\right.$ and $N(Q)=$ $\mathcal{S}\}$.

Then

$$
\min _{Q \in \mathcal{A}} Q^{*} Q=P_{0}^{*} P_{0}
$$

where $P_{0}=P_{\mathcal{M}^{\perp} \ominus \mathcal{S} / / \mathcal{S}}$. Moreover, $P_{0}$ is the unique projection in $\mathcal{A}$ satisfying (4).

Proof: Observe that $\mathcal{S}^{\perp} \cap \mathcal{M}=\left(\mathcal{S}+\mathcal{M}^{\perp}\right)^{\perp}=\{0\}$ and consider the projection $P_{\mathcal{M}}$. Then $P_{\mathcal{M}} \geq 0$ and $\left(P_{\mathcal{M}} \mathcal{S}\right)^{\perp}=P_{\mathcal{M}}^{-1}\left(\mathcal{S}^{\perp}\right)=P_{\mathcal{M}}^{-1}\left(\mathcal{S}^{\perp} \cap\right.$ $\mathcal{M})=N\left(P_{\mathcal{M}}\right)=\mathcal{M}^{\perp}$. Therefore, by Theorem 3.2 the pair $\left(P_{\mathcal{M}}, \mathcal{S}\right)$ is compatible. Observe that, by Krein's lemma, $Q \in \mathcal{A}$ if and only if $I-Q \in \mathcal{P}\left(P_{\mathcal{M}}, \mathcal{S}\right)$.

Applying Theorem 5.1, for every $E \in \mathcal{P}\left(P_{\mathcal{M}}, \mathcal{S}\right)$ it holds that $\|(I-$ $\left.P_{\mathcal{M}, \mathcal{S}}\right) h\|\leq\|(I-E) h \|$, for $h \in \mathcal{H}$. Or equivalently, $P_{0}^{*} P_{0} \leq Q^{*} Q$, where $P_{0}=I-P_{\mathcal{M}, \mathcal{S}}$ and $Q=I-E$. Finally, observe that, by the definition of $P_{\mathcal{M}, \mathcal{S}}$ it follows that $R\left(P_{0}\right)=\mathcal{M}^{\perp} \ominus \mathcal{S}$ and $N\left(P_{0}\right)=\mathcal{S}$.

To see the uniqueness, suppose that $P_{1} \in \mathcal{A}$ verifies that $\min _{Q \in \mathcal{A}} Q^{*} Q=$ $P_{1}^{*} P_{1}$. Then, $P_{1}^{*} P_{1}=P_{0}^{*} P_{0}$. Using Proposition 3.8 we can write $P_{1}=$ $P_{0}+W$, where $W$ is an operator in $L(\mathcal{H})$ such that $R(W) \subseteq \mathcal{S} \cap \mathcal{M}^{\perp}$ and $\mathcal{S} \subseteq N(W)$. Therefore, $P_{0}^{*} P_{0}=P_{0}^{*} P_{0}-P_{0}^{*} W-W^{*} P_{0}+W^{*} W$. Observe that $P_{0}^{*} W=W^{*} P_{0}=0$, because $R(W) \subseteq N\left(P_{0}^{*}\right)=M+\mathcal{S} \cap \mathcal{M}^{\perp}$. Then, $W^{*} W=0$, so that $W=0$ and $P_{1}=P_{0}$.

In this case, the symmetric problem is the following: suppose that $\mathcal{M}$ and $\mathcal{S}$ are two closed subspaces of $\mathcal{H}$ such that $\mathcal{M} \cap \mathcal{S}^{\perp}=\{0\}$ and $\mathcal{M}+\mathcal{S}^{\perp}$ is a proper closed subspace of $\mathcal{H}$; consider the set $\mathcal{A}^{*}=\{Q \in$ $\mathcal{Q}: R(Q)=\mathcal{S}^{\perp}$ and $\left.\mathcal{M} \subseteq N(Q)\right\}$.

Then

$$
\min _{Q \in \mathcal{A}^{*}} Q Q^{*}=Q_{0}^{*} Q_{0},
$$

where $Q_{0}=P_{\mathcal{S}^{\perp} / / \mathcal{M}+\left(\mathcal{S} \cap \mathcal{M}^{\perp}\right)}$; moreover $Q_{0}$ is the unique projection satisfying (5).

Corollary 5.3. Let $A \in L(\mathcal{H})^{+}$and $\mathcal{S}$ a closed subspace of $\mathcal{H}$ such that $(A, \mathcal{S})$ is compatible. Then

$$
\min _{Q \in \mathcal{P}(A, \mathcal{S})}\left(I-Q^{*}\right)(I-Q)=\left(I-P_{A, \mathcal{S}}\right)^{*}\left(I-P_{A, \mathcal{S}}\right) .
$$

Moreover, the projection $I-P_{A, \mathcal{S}}$ is the unique solution to this problem. 
Remark 5.4. If, instead of problem (2), we consider the problem of minimizing $Q^{*} A Q$ among all the projections with kernel $\mathcal{S}\left(A \in L(\mathcal{H})^{+}\right.$is fixed), then we obtain the following result [13,4.2 and 4.3]): the minimum is attained if and only if $(A, \mathcal{S})$ is compatible; in such case, the minimum coincides with $A(I-E)$, for any $E \in \mathcal{P}(A, \mathcal{S})$. It is called the shorted operator of $A$ to $\mathcal{S}$; see $[\mathbf{1 3}]$ for details.

\section{Interpolating and smoothing problems}

Of course, orthogonal projections and Moore-Penrose inverses, and their relatives, appear naturally when different least squares problems are solved. This is the case here. In this section we study classical interpolating and smoothing problems where the oblique projections and compatibility hypothesis play an important role. These problems were introduced and studied by Atteia [5], and they are known as "abstract spline problems". Throughout this section, we consider $T \in L(\mathcal{H}, \mathcal{E})$ and $V \in L(\mathcal{H}, \mathcal{F})$ such that $V$ is surjective; we write $A:=T^{*} T, \mathcal{S}:=N(V)$ and $\mathcal{N}=\mathcal{S} \cap N(T)$.

\section{Interpolating problems.}

Consider the following minimization problem

$$
\arg \min \|T h\|, \quad \text { subject to } V h=f_{0},
$$

where $f_{0} \in \mathcal{F}$. This is known as an interpolating spline problem. We denote

$$
\operatorname{spl}\left(T, \mathcal{S}, f_{0}\right):=\left\{h_{0} \in \mathcal{H}: V h_{0}=f_{0},\left\|T h_{0}\right\|=\min _{V h=f_{0}}\|T h\|\right\} .
$$

Atteia [6] (see also the surveys by Champion, Lenard and Mills [10] and [11]) proved that if $R(T)$ and $T \mathcal{S}$ are closed and $\mathcal{N}=\{0\}$ then there exists a unique solution $h_{0}$ of (6). In this case, $A h_{0} \in \mathcal{S}^{\perp}$. The following theorem, proved in [14], generalizes these results and characterizes the set of solutions of (6). Observe that the Dixmier angle condition is weaker than the closed range hypothesis usually used.

Proposition 6.1. The following conditions are equivalent:

(1) Problem (6) admits a solution for every $f_{0} \in \mathcal{F}$;

(2) $c_{0}\left(\overline{A \mathcal{S}}, \mathcal{S}^{\perp}\right)<1$.

If any (and then both) of these conditions hold, then

$$
\operatorname{spl}\left(T, \mathcal{S}, f_{0}\right)=\left\{(I-Q) V^{\dagger} f_{0}: Q \in \mathcal{P}(A, \mathcal{S})\right\} .
$$

In particular, if $\mathcal{N}=\{0\}, \operatorname{spl}\left(T, \mathcal{S}, f_{0}\right)=\left\{\left(I-P_{A, \mathcal{S}}\right) V^{\dagger} f_{0}\right\}$. 
The same result holds replacing $V^{\dagger}$ by any pseudoinverse $V^{\prime}$ of $V$. If $h_{0} \in \operatorname{spl}\left(T, \mathcal{S}, f_{0}\right)$ then $A h_{0} \in \mathcal{S}^{\perp}$. Moreover, $h_{0}=\left(I-P_{A, \mathcal{S}}\right) V^{\dagger} f_{0}$ is the unique vector in $\operatorname{spl}\left(T, \mathcal{S}, f_{0}\right)$ with minimal norm.

Consider $Q \in P(A, \mathcal{S})$ and define $V_{Q}^{\prime}=(I-Q) V^{\dagger}$. It is easy to see that $V_{Q}^{\prime}$ verifies $V V_{Q}^{\prime} V=V, V_{Q}^{\prime} V V_{Q}^{\prime}=V_{Q}^{\prime}, V_{Q}^{\prime} V=I-Q$ and $V V_{Q}^{\prime}=I_{\mathcal{F}}$. Observe that $R\left(V_{Q}^{\prime}\right)=N(Q)$ and that $V_{Q}^{\prime}$ is a generalized pseudoinverse, corresponding to the decomposition $\mathcal{H}=N(Q) \dot{+} R(Q)$ (since $V$ is surjective, no decomposition of $\mathcal{H}$, associated to range of $V$, is needed). Define $C: \mathcal{F} \rightarrow \mathcal{H}$ by $C f_{0}:=h_{0}=\left(I-P_{A, \mathcal{S}}\right) V^{\dagger} f_{0}$. Then $C=\left(I-P_{A, \mathcal{S}}\right) V^{\dagger}=V_{P_{A, \mathcal{S}}}^{\prime}$. It follows easily that

$$
\operatorname{spl}\left(T, \mathcal{S}, f_{0}\right)=V_{P_{A, \mathcal{S}}}^{\prime} f_{0}+\mathcal{N}=\left(I-P_{A, \mathcal{S}}\right) V^{\dagger} f_{0}+\mathcal{N} .
$$

An operator $C \in L(\mathcal{H})$ is a $(T, \mathcal{S})$-spline operator if $C h \in \operatorname{spl}(T, \mathcal{S}, V h)$, for every $h \in \mathcal{H}$. This notion was defined by Izumino $[\mathbf{2 8}]$ who gave a characterization of these operators, for a closed range operator $T$. Using the formula for $P_{A, \mathcal{S} \ominus \mathcal{N}}$ proved in Theorem 4.6, we are able to generalize Izumino's result.

Proposition 6.2. Suppose that $c_{0}\left(\overline{A \mathcal{S}}, \mathcal{S}^{\perp}\right)<1$ and let $C \in L(\mathcal{H})$. Then $C$ is a $(T, \mathcal{S})$-spline operator if and only if $C=I-\left(T P_{\mathcal{S}}\right)^{\dagger} T+W=$ $I-P_{A, \mathcal{S} \ominus \mathcal{N}}+W$, where $W \in L(\mathcal{H})$ and $R(W) \subseteq \mathcal{N}$.

Proof: The condition $c_{0}\left(\overline{A \mathcal{S}}, \mathcal{S}^{\perp}\right)<1$ guarantees the existence of solution of the interpolating problem for every $f \in \mathcal{F}$ and also the existence of the projection $P_{A, \mathcal{S}}$. If $C \in L(\mathcal{H})$ is a $(T, \mathcal{S})$-spline operator then $C h \in \operatorname{spl}(T, \mathcal{S}, V h)$, for every $h \in \mathcal{H}$. Therefore, by the formula above, $C h=\left(I-P_{A, \mathcal{S}}\right) V^{\dagger} V h+w=\left(I-P_{A, \mathcal{S}}\right) h+w$, with $w \in \mathcal{N}$, because $\left(I-P_{A, \mathcal{S}}\right) V^{\dagger} V h=\left(I-P_{A, \mathcal{S}}\right) P_{\mathcal{S}^{\perp}} h=\left(I-P_{A, \mathcal{S}}\right) h$ since $\left(I-P_{A, \mathcal{S}}\right) P_{\mathcal{S}}=0$. But $P_{A, \mathcal{S}}=P_{A, \mathcal{S} \ominus \mathcal{N}}+P_{\mathcal{N}}$, so that $C h=\left(I-P_{A, \mathcal{S} \ominus \mathcal{N}}\right) h+w^{\prime}$, with $w^{\prime} \in \mathcal{N}$. Applying Proposition 4.6, $P_{A, \mathcal{S} \ominus \mathcal{N}}=\left(T P_{\mathcal{S}}\right)^{\dagger} T$, hence, $C=$ $I-\left(T P_{\mathcal{S}}\right)^{\dagger} T+W$, where $R(W) \subseteq \mathcal{N}$. The converse is immediate.

\section{Smoothing problems.}

We use the same notations as before. Consider the following problem

$$
\arg \min \left(\|T h\|^{2}+\rho\left\|V h-f_{0}\right\|^{2}\right), \quad \text { for } h \in \mathcal{H},
$$

where $f_{0} \in \mathcal{F}$ and $\rho>0$. In the literature, this is known as a smoothing problem. The choice of the parameter $\rho$ is the subject of an extensive bibliography. This is closely related to Tikhonov regularization techniques (see $[\mathbf{2 3}]$ ). 
Define $K: \mathcal{H} \rightarrow \mathcal{E} \times \mathcal{F}, K h=(T h, V h)$, for $h \in \mathcal{H}$. Consider $\mathcal{F}$ with the inner product $\langle,\rangle_{\rho}=\rho\langle$,$\rangle and \mathcal{E} \times \mathcal{F}$ with the associated inner product

$$
\left\langle(e, f),\left(e^{\prime}, f^{\prime}\right)\right\rangle=\left\langle e, e^{\prime}\right\rangle+\rho\left\langle f, f^{\prime}\right\rangle, \text { for } e, e^{\prime} \in \mathcal{H}, f, f^{\prime} \in \mathcal{F} .
$$

Observe that problem (7) can be restated as

$$
\arg \min \left\|K h-\left(0, f_{0}\right)\right\|, \quad \text { for } h \in \mathcal{H},
$$

where $\|(e, f)\|^{2}=\langle e, e\rangle^{2}+\rho\langle f, f\rangle^{2}$, for all $(e, f) \in \mathcal{E} \times \mathcal{F}$.

Lemma 6.3. If $c_{0}\left(\overline{A \mathcal{S}}, \mathcal{S}^{\perp}\right)<1$, then

$$
R(K)=T \mathcal{S} \times\{0\} \oplus\left\{\left(T\left(I-P_{A, \mathcal{S}}\right) V^{\dagger} f, f\right): f \in \mathcal{F}\right\} .
$$

Proof: By Theorem 3.2, the pair $(A, \mathcal{S})$ is compatible. Let $Q=P_{A, \mathcal{S}}$ and decompose $\mathcal{H}=\mathcal{S} \dot{+} N(Q)$; then $R(K)=K \mathcal{S}+K N(Q)$. Observe that $K \mathcal{S}=T \mathcal{S} \times\{0\}$ because $V s=0$, for $s \in \mathcal{S}$.

In order to compute $K N(Q)$ observe that $V N(Q)=V V_{Q}^{\prime}(\mathcal{F})=\mathcal{F}$ because $V$ is surjective. Then $K N(Q)=\{(T h, V h): h \in N(Q)\}=$ $\left\{\left(T V_{Q}^{\prime} f, f\right): f \in \mathcal{F}\right\}$. To see that the sum is orthogonal consider $(T s, 0) \in T \mathcal{S} \times\{0\}$ and $\left(T V_{Q}^{\prime} f, f\right)$, for $f \in \mathcal{F}$; then $\left\langle(T s, 0),\left(T V_{Q}^{\prime} f, f\right)\right\rangle=$ $\left\langle T s, T V_{Q}^{\prime} f\right\rangle_{\mathcal{E}}=\left\langle T^{*} T s, V_{Q}^{\prime} f\right\rangle_{\mathcal{E}}=\left\langle A s, V_{Q}^{\prime} f\right\rangle_{\mathcal{E}}=0$ because $V_{Q}^{\prime} f \in N(Q) \subseteq$ $(A \mathcal{S})^{\perp}$.

Theorem 6.4. If $c_{0}\left(\overline{A \mathcal{S}}, \mathcal{S}^{\perp}\right)<1$ then the set of solutions of problem (7) is $\operatorname{spl}(T, \mathcal{S}, \tilde{f})$, where $\tilde{f}=\left(I+\frac{1}{\rho} V_{Q}^{\prime *} A V_{Q}^{\prime}\right)^{-1} f_{0}$ and $Q=P_{A, \mathcal{S}}$.

Proof: When $f_{0}=0$, the set of solutions of (7) or (8) is $N(K)=N(T) \cap$ $N(V)$. In this case $\tilde{f}=0$ so that $\operatorname{spl}(T, \mathcal{S}, 0)=N(T) \cap N(V)$.

Suppose that $f_{0} \neq 0$ and let $Q=P_{A, \mathcal{S}}$; if $h \in \mathcal{H}, h=Q h+(I-$ $Q) h=s+V_{Q}^{\prime} f$, with $s=Q h \in \mathcal{S}$ and $f=V h \in \mathcal{F}$ (observe that $V_{Q}^{\prime} f=V_{Q}^{\prime} V h=(I-Q) h$ because $\left.V_{Q}^{\prime} V=I-Q\right)$.

Then $\left\|K h-\left(0, f_{0}\right)\right\|^{2}=\left\|(T s, 0)+\left(T V_{Q}^{\prime} f, f-f_{0}\right)\right\|^{2}=\|(T s, 0)\|^{2}+$ $\left\|\left(T V_{Q}^{\prime} f, f-f_{0}\right)\right\|^{2}=\|T s\|^{2}+\left\|\left(T V_{Q}^{\prime} f, f-f_{0}\right)\right\|^{2}$ because the sets $T \mathcal{S} \times\{0\}$ and $\left\{\left(T V_{Q}^{\prime} f, f\right): f \in \mathcal{F}\right\}$ are orthogonal, by Lemma 6.3. Therefore,

$$
\begin{aligned}
\min _{h \in \mathcal{H}}\left\|K h-\left(0, f_{0}\right)\right\|^{2} & =\min _{s \in \mathcal{S}}\|T s\|^{2}+\min _{f \in \mathcal{F}}\left\|\left(T V_{Q}^{\prime} f, f-f_{0}\right)\right\|^{2} \\
& =\min _{f \in \mathcal{F}}\left\|\left(T V_{Q}^{\prime} f, f-f_{0}\right)\right\|^{2} .
\end{aligned}
$$

Observe that

$\left.\min _{f \in \mathcal{F}}\left\|\left(T V_{Q}^{\prime} f, f-f_{0}\right)\right\|=\min _{f \in \mathcal{F}} \|\left(f, T V_{Q}^{\prime} f\right)-\left(f_{0}, 0\right)\right) \|_{\mathcal{F} \times \mathcal{E}}=d\left(\left(f_{0}, 0\right), \Gamma\left(T V_{Q}^{\prime}\right)\right)$, 
where $\Gamma(C)$ denotes the graph of the operator $C$ and $d(P, \mathcal{M})$ denotes the distance from the point $P$ to the set $\mathcal{M}$. Since $T V_{Q}^{\prime}$ is bounded $\Gamma\left(T V_{Q}^{\prime}\right)$ is closed so that $d\left(\left(f_{0}, 0\right), \Gamma\left(T V_{Q}^{\prime}\right)\right)=\left\|(I-P)\left(f_{0}, 0\right)\right\|$, where $P$ is the orthogonal projection onto $\Gamma\left(T V_{Q}^{\prime}\right)$.

In order to compute the projection $P$, recall that if $C \in L(\mathcal{K})$, for a Hilbert space $\mathcal{K}$; then $\Gamma(C)^{\perp}=U\left(\Gamma\left(C^{*}\right)\right)$, where $U(x, y)=(-y, x)$. In this case, observe that $V_{Q}^{\prime}:\left(\mathcal{F},\langle,\rangle_{\rho}\right) \rightarrow(\mathcal{H},\langle\rangle$,$) . Then, the adjoint$ operator of $V_{Q}^{\prime}$ is $\frac{1}{\rho} V_{Q}^{\prime *}$, where $V_{Q}^{\prime *}$ is the adjoint of $V_{Q}^{\prime}:(\mathcal{F},\langle\rangle,) \rightarrow$ $(\mathcal{H},\langle\rangle$,$) . Therefore,$

$$
\Gamma\left(T V_{Q}^{\prime}\right)^{\perp}=\left\{\left(-\frac{1}{\rho} V_{Q}^{\prime *} T^{*} e, e\right): e \in \mathcal{E}\right\} .
$$

Given $\left(f_{0}, 0\right)$, write $\left(f_{0}, 0\right)=P\left(f_{0}, 0\right)+(I-P)\left(f_{0}, 0\right)$ with

$$
P\left(f_{0}, 0\right)=\left(\tilde{f}, T V_{Q}^{\prime} \tilde{f}\right) \quad \text { and } \quad(I-P)\left(f_{0}, 0\right)=\left(-\frac{1}{\rho} V_{Q}^{\prime *} T^{*} \tilde{e}, \tilde{e}\right)
$$

for unique $\tilde{f} \in \mathcal{F}$ and $\tilde{e} \in \mathcal{E}$. Then,

$$
f_{0}=\tilde{f}-\frac{1}{\rho} V_{Q}^{\prime *} T^{*} \tilde{e} \quad \text { and } \quad 0=T V_{Q}^{\prime} \tilde{f}+\tilde{e} .
$$

Therefore $f_{0}=\tilde{f}+\frac{1}{\rho} V_{Q}^{\prime *} T^{*} T V_{Q}^{\prime} \tilde{f}=\left(I+\frac{1}{\rho} V_{Q}^{\prime *} A V_{Q}^{\prime}\right) \tilde{f}$. If $B=\frac{1}{\rho} V_{Q}^{\prime *} A V_{Q}^{\prime}$ then $B \geq 0$ so that $I+B$ is strictly positive. Therefore $f_{0}=(I+B) \tilde{f}$, or $\tilde{f}=(I+B)^{-1} f_{0}$. Hence $P\left(f_{0}, 0\right)=\left(\tilde{f}, T V_{Q}^{\prime} \tilde{f}\right)$ and

$$
\min _{h \in \mathcal{H}}\left\|K h-\left(0, f_{0}\right)\right\|=\left\|(I-P)\left(f_{0}, 0\right)\right\| .
$$

We claim that $h_{0} \in \mathcal{H}$ verifies that $\left\|K h_{0}-\left(0, f_{0}\right)\right\|=\min _{h \in \mathcal{H}} \| K h-$ $\left(0, f_{0}\right) \|$ if and only if there exists $E \in \mathcal{P}(A, \mathcal{S})$ such that $h_{0}=(I-$ $E) V^{\dagger} \tilde{f}$. To prove this assertion suppose that $h_{0}=(I-E) V^{\dagger} \tilde{f}$, for $E \in \mathcal{P}(A, \mathcal{S})$. Then $K h_{0}=\left(T(I-E) V^{\dagger} \tilde{f}, V(I-E) V^{\dagger} \tilde{f}\right)=(T(I-$ $\left.Q) V^{\dagger} \tilde{f}, \tilde{f}\right)=\left(T V_{Q}^{\prime} \tilde{f}, \tilde{f}\right)$ (because, by Proposition 4.3, $E=Q+W$, with $R(W) \subseteq N(T) \cap \mathcal{S}$ and $\mathcal{S} \subseteq N(W))$, so that $T(I-E)=T(I-Q))$. Therefore $\left\|K h_{0}-\left(0, f_{0}\right)\right\|=\left\|(I-P)\left(f_{0}, 0\right)\right\|=\min _{h \in \mathcal{H}}\left\|K h-\left(0, f_{0}\right)\right\|$.

Conversely, suppose that $h_{0} \in \mathcal{H}$ verifies that $\left\|K h_{0}-\left(0, f_{0}\right)\right\|=$ $\min _{h \in \mathcal{H}}\left\|K h-\left(0, f_{0}\right)\right\|$. Then, as before, there exist unique $s \in \mathcal{S}$ and $f^{\prime} \in \mathcal{F}$ such that $h_{0}=s+V_{Q}^{\prime} f^{\prime}$. Therefore,

$$
\left\|K h_{0}-\left(0, f_{0}\right)\right\|^{2}=\|T s\|^{2}+\left\|\left(T V_{Q}^{\prime} f^{\prime}, f^{\prime}-f_{0}\right)\right\|^{2} .
$$


Hence,

$$
\begin{aligned}
\|T s\|^{2}+\left\|\left(T V_{Q}^{\prime} f^{\prime}, f^{\prime}-f_{0}\right)\right\|^{2} & =\min _{h \in \mathcal{H}}\left\|K h-\left(0, f_{0}\right)\right\|^{2} \\
& =\min _{f \in \mathcal{F}}\left\|\left(T V_{Q}^{\prime} f, f-f_{0}\right)\right\|^{2}=\left\|\left(T V_{Q}^{\prime} \tilde{f}, \tilde{f}-f_{0}\right)\right\|^{2} .
\end{aligned}
$$

But since $\left\|\left(T V_{Q}^{\prime} \tilde{f}, \tilde{f}-f_{0}\right)\right\| \leq\left\|\left(T V_{Q}^{\prime} f, f-f_{0}\right)\right\|$, for all $f \in \mathcal{F}$, in particular for $f^{\prime}$, it follows that $\|T s\|=0$ and $\left\|\left(T V_{Q}^{\prime} \tilde{f}, \tilde{f}-f_{0}\right)\right\|=$ $\left\|\left(T V_{Q}^{\prime} f^{\prime}, f^{\prime}-f_{0}\right)\right\|=\left\|(I-P)\left(f_{0}, 0\right)\right\|$. From the last equality it follows that $f^{\prime}=\tilde{f}$. Then $s \in N(T) \cap \mathcal{S}$ and $h_{0}=s+V_{Q}^{\prime} \tilde{f}$. Define $W \in L\left(\mathcal{S}^{\perp}, N(T) \cap \mathcal{S}\right.$ ) such that $W V^{\dagger} \tilde{f}=-s$, (observe that this is possible because $\left.V^{\dagger} \tilde{f} \neq 0\right)$. Then, $E=Q+W \in \mathcal{P}(A, \mathcal{S})$ and $(I-E) V^{\dagger} \tilde{f}=V_{Q}^{\prime} \tilde{f}-W V^{\dagger} \tilde{f}=V_{Q}^{\prime} \tilde{f}+s=h_{0}$.

\section{Mixed problems.}

These are problems which can be splitted in two parts, one on interpolating splines and the other on smoothing splines (see the book [8]). From now on let $\mathcal{H}, \mathcal{E}, \mathcal{F}_{1}$ and $\mathcal{F}_{2}$ be Hilbert spaces. Consider $T \in$ $L(\mathcal{H}, \mathcal{E}), V_{i} \in L\left(\mathcal{H}, \mathcal{F}_{i}\right)$ such that $V_{i}$ has closed range $(i=1,2)$ and $\rho>0$. Denote $A:=T^{*} T, \mathcal{S}_{i}:=N\left(V_{i}\right)(i=1,2)$ and $\mathcal{S}:=\mathcal{S}_{1} \cap \mathcal{S}_{2}$; we suppose that $c_{0}\left(\overline{A \mathcal{S}}, \mathcal{S}^{\perp}\right)<1$ and denote $Q:=P_{A, \mathcal{S}}$. Consider the following problem

$$
\arg \min \left(\|T h\|^{2}+\rho\left\|V_{2} h-f_{2}\right\|^{2}\right), \quad \text { subject to } V_{1} h=f_{1},
$$

where $f_{1} \in R\left(V_{1}\right)$ and $f_{2} \in \mathcal{F}_{2}$.

As in the smoothing problem, define the operator $K: \mathcal{H} \rightarrow \mathcal{E} \times \mathcal{F}_{2}$,

$$
K h=\left(T h, V_{2} h\right),
$$

with $\|(e, f)\|^{2}=\|e\|^{2}+\rho\|f\|^{2}$, for $e \in \mathcal{E}$ and $f \in \mathcal{F}_{2}$ (or equivalently we consider $\left(\mathcal{F}_{2},\langle,\rangle_{\rho}\right)$, where $\left.\langle,\rangle_{\rho}=\rho\langle\rangle,\right)$. Then, problem (9) is equivalent to

$$
\arg \min \left\|K h-\left(0, f_{2}\right)\right\|^{2}, \quad \text { subject to } V_{1} h=f_{1} .
$$

Theorem 6.5. If $c_{0}\left(\overline{A \mathcal{S}}, \mathcal{S}^{\perp}\right)<1$ and $\mathcal{S}_{1}+\mathcal{S}_{2}$ is closed then problem (9) has a solution. Moreover, the set of solutions of (9) is given by

$$
\operatorname{spl}\left(T, \mathcal{S},\left(f_{1}, P_{\mathcal{M}_{2}} \tilde{f}+V_{2} V_{1}^{\dagger} f_{1}\right)\right),
$$

where $\mathcal{S}=N(V)$, with $V=\left(V_{1}, V_{2}\right) \in L\left(\mathcal{H}, \mathcal{F}_{1} \times \mathcal{F}_{2}\right)$ and $\tilde{f}$ is as in Lemma 6.8 . 
Instead of presenting the complete proof, we describe the main steps in a series of lemmas. In fact, these lemmas and the proof of Theorem 6.4 lead to a proof of Theorem 6.5. The common hypothesis for these lemmas is that $c_{0}\left(\overline{A \mathcal{S}}, \mathcal{S}^{\perp}\right)<1$ and $\mathcal{S}_{1}+\mathcal{S}_{2}$ is closed.

Lemma 6.6. (1) The subspaces $\mathcal{M}_{1}=(I-Q) \mathcal{S}_{1}$ and $\mathcal{M}_{2}=V_{2} \mathcal{S}_{1}$ are closed;

(2) $\left(\left.T V_{2}\right|_{\mathcal{M}_{1}}\right)^{-1}: \mathcal{M}_{2} \rightarrow \mathcal{M}_{1}$ is an isomorphism;

(3) $\left.K \mathcal{S}_{1}=T \mathcal{S} \times\{0\} \oplus\left\{\left(\left.T V_{2}\right|_{\mathcal{M}_{1}}\right)^{-1} r, r\right): r \in \mathcal{M}_{2}\right\}$.

Lemma 6.7. If $\left(e_{0}, f_{0}\right)=\left(0, f_{2}\right)-K(I-Q) V_{1}^{\dagger} f_{1}$. Then

$$
\min _{V_{1} h=f_{1}}\left\|K h-\left(0, f_{2}\right)\right\|=d\left(\left(f_{0}, e_{0}\right), \Gamma\left(T\left(\left.V_{2}\right|_{\mathcal{M}_{1}}\right)^{-1}\right)\right) .
$$

Lemma 6.8. Consider $P: \mathcal{F}_{2} \times \mathcal{E} \rightarrow \mathcal{F}_{2} \times \mathcal{E}$ the orthogonal projection onto $\Gamma\left(T\left(\left.V_{2}\right|_{\mathcal{M}_{1}}\right)^{-1}\right)$. Then

$$
P\left(f_{0}, e_{0}\right)=\left(P_{\mathcal{M}_{2}} \tilde{f}, T\left(V_{2} P_{\mathcal{M}_{1}}\right)^{\dagger} \tilde{f}\right),
$$

where $B=I+\frac{1}{\rho}\left(V_{2} P_{\mathcal{M}_{1}}\right)^{* \dagger} A\left(V_{2} P_{\mathcal{M}_{1}}\right)^{\dagger}, \tilde{f}=B^{-1}\left(f_{2}-\left(V_{2}+\frac{1}{\rho}\left(V_{2} P_{\mathcal{M}_{1}}\right)^{* \dagger} A\right) h_{1}\right)$, $\left(f_{0}, e_{0}\right)$ is defined as in Lemma 6.7 and $h_{1}=(I-Q) V_{1}^{\dagger} f_{1}$.

Acknowledgement. We would like to thank Jorge Antezana for his comments and for providing us Example 3.7.

\section{References}

[1] W. N. Anderson, Jr. and G. E. Trapp, Shorted operators. II, SIAM J. Appl. Math. 28 (1975), 60-71.

[2] E. Andruchow, G. Corach, and D. Stojanoff, Geometry of oblique projections, Studia Math. 137(1) (1999), 61-79.

[3] J. Antezana and G. Corach, Sampling theory, oblique projections and a question by Smale and Zhou, Appl. Comput. Harmon. Anal. 21(2) (2006), 245-253.

[4] M. L. Arias, G. Corach, and M. C. González, Generalized inverses and Douglas equations, Proc. Amer. Math. Soc. 136(9) (2008), 3177-3183.

[5] M. Atтeia, Généralisation de la définition et des propriétés des "spline fonctions", C. R. Acad. Sci. Paris 260 (1965), 3550-3553.

[6] M. Aтteia, "Hilbertian kernels and spline functions", Studies in Computational Mathematics 4, North-Holland Publishing Co., Amsterdam, 1992. 
[7] A. Ben-Israel and T. N. E. Greville, "Generalized inverses. Theory and applications", Second edition, CMS Books in Mathematics/Ouvrages de Mathématiques de la SMC 15, Springer-Verlag, New York, 2003.

[8] A. Yu. Bezhaev and A. V. Vasilenko, "Variational theory of splines", Kluwer Academic/Plenum Publishers, New York, 2001.

[9] C. DE Boor, Convergence of abstract splines, J. Approx. Theory 31(1) (1981), 80-89.

[10] R. Champion, C. T. Lenard, and T. M. Mills, An introduction to abstract splines, Math. Sci. 21(1) (1996), 8-26.

[11] R. Champion, C. T. Lenard, and T. M. Mills, A variational approach to splines, Papers in honour of David Elliott on the occasion of his sixty-fifth birthday, ANZIAM J. 42(1) (2000), 119-135.

[12] G. Corach, J. I. Giribet, and A. Maestripieri, Sard's approximation processes and oblique projections, Studia Math. 194(1) (2009), 65-80.

[13] G. Corach, A. Maestripieri, And D. Stojanoff, Oblique projections and Schur complements, Acta Sci. Math. (Szeged) 67(1-2) (2001), 337-356.

[14] G. Corach, A. Maestripieri, and D. Stojanoff, Oblique projections and abstract splines, J. Approx. Theory 117(2) (2002), 189-206.

[15] G. Corach, A. Maestripieri, and D. Stojanoff, A classification of projectors, in: "Topological algebras, their applications, and related topics", Banach Center Publ. 67, Polish Acad. Sci., Warsaw, 2005, pp. 145-160.

[16] F. Deutsch, The angle between subspaces of a Hilbert space, in: "Approximation theory, wavelets and applications" (Maratea, 1994), NATO Adv. Sci. Inst. Ser. C Math. Phys. Sci. 454, Kluwer Acad. Publ., Dordrecht, 1995, pp. 107-130.

[17] F. Deutsch, "Best approximation in inner product spaces", CMS Books in Mathematics/Ouvrages de Mathématiques de la SMC 7, Springer-Verlag, New York, 2001.

[18] J. Dixmier, Position relative de deux variétés linéaires fermées dans un espace de Hilbert, Revue Sci. 86 (1948), 387-399.

[19] R. G. Douglas, On majorization, factorization, and range inclusion of operators on Hilbert space, Proc. Amer. Math. Soc. 17 (1966), 413-415.

[20] Y. C. EldAR, Sampling with arbitrary sampling and reconstruction spaces and oblique dual frame vectors, J. Fourier Anal. Appl. 9(1) (2003), 77-96. 
[21] K. Friedrichs, On certain inequalities and characteristic value problems for analytic functions and for functions of two variables, Trans. Amer. Math. Soc. 41(3) (1937), 321-364.

[22] G. H. Golub and C. F. VAn LoAn, "Matrix computations", Third edition, Johns Hopkins Studies in the Mathematical Sciences, Johns Hopkins University Press, Baltimore, MD, 1996.

[23] P. C. HANSEn, "Rank-deficient and discrete ill-posed problems", Numerical aspects of linear inversion, SIAM Monographs on Mathematical Modeling and Computation, Society for Industrial and Applied Mathematics (SIAM), Philadelphia, PA, 1998.

[24] D. A. HARVILle, "Matrix algebra from a statistician's perspective", Springer-Verlag, New York, 1997.

[25] S. Hassi and K. NordSTRÖM, On projections in a space with an indefinite metric, Linear Algebra Appl. 208/209 (1994), 401-417.

[26] M. R. Hestenes And E. Stiefel, Methods of conjugate gradients for solving linear systems, J. Research Nat. Bur. Standards $\mathbf{4 9}$ (1952), 409-436 (1953).

[27] A. Hirabayashi and M. Unser, Consistent sampling and signal recovery, IEEE Trans. Signal Process. 55(8) (2007), 4104-4115.

[28] S. Izumino, Convergence of generalized inverses and spline projectors, J. Approx. Theory 38(3) (1983), 269-278.

[29] T. Kato, "Perturbation theory for linear operators", Reprint of the 1980 edition, Classics in Mathematics, Springer-Verlag, Berlin, 1995.

[30] M. Krein, The theory of self-adjoint extensions of semi-bounded Hermitian transformations and its applications. I, (Russian), Rec. Math. [Mat. Sbornik] N.S. 20(62) (1947). 431-495.

[31] C. Lanczos, An iteration method for the solution of the eigenvalue problem of linear differential and integral operators, J. Research Nat. Bur. Standards 45 (1950), 255-282.

[32] N. Minamide and K. NaKamura, A restricted pseudoinverse and its application to constrained minima, SIAM J. Appl. Math. 19 (1970), 167-177.

[33] M. Z. NASHED, Inner, outer, and generalized inverses in Banach and Hilbert spaces, Numer. Funct. Anal. Optim. 9(3-4) (1987), $261-325$.

[34] N. K. NiKOLSKI, "Operators, functions, and systems: an easy reading", Vol. 1: Hardy, Hankel, and Toeplitz, Mathematical Surveys and Monographs 92. Vol. 2: Model operators and systems, Mathematical Surveys and Monographs 93. Translated from the French by 
Andreas Hartmann, American Mathematical Society, Providence, RI, 2002.

[35] Z. Pasternak-Winiarski, On the dependence of the orthogonal projector on deformations of the scalar product, Studia Math. 128(1) (1998), 1-17.

[36] È. L. PeKarev, Shorts of operators and some extremal problems, Acta Sci. Math. (Szeged) 56(1-2) (1992), 147-163.

[37] V. PTÁk, Extremal operators and oblique projections, (Russian), Časopis Pěst. Mat. 110(4) (1985), 343-350, 413.

[38] A. SARd, Approximation and variance, Trans. Amer. Math. Soc. 73 (1952), 428-446.

[39] B. Shekhtman, Unconditional convergence of abstract splines, $J$. Approx. Theory 30(3) (1980), 237-246.

[40] M. Unser and A. Aldroubi, A general sampling theory for nonideal adquisition devices, IEEE Trans. Signal Process. 42(11) (1994), 2915-2925.

Departamento de Matemática

Facultad de Ingeniería, UBA and

Instituto Argentino de Matemática - CONICET

Saavedra 15

Buenos Aires (1083)

Argentina

E-mail address: gcorach@fi.uba.ar

E-mail address: amaestri@fi.uba.ar

Primera versió rebuda el 29 de juliol de 2009, darrera versió rebuda el 8 d'abril de 2010 . 